\title{
Current New Applications of Laser Plasmas
}

\author{
Allan A. Hauer
}

David W. Forslund

Colin J. McKinstrie*

Justin S. Wark**

Philip J. Hargis, Jr. ${ }^{\dagger}$

Roy A. Hamil ${ }^{t}$

Joseph M. Kindel ${ }^{+t}$

\section{DISCLAIMER}

This report was prepared as an account of work sponsored by an agency of the United States Government. Neither the United States Government nor any agency thereof, nor any of their employees, makes any warranty, express or implied, or assumes any legal liability or responsibility for the accuracy, completeness, or usefulness of any information, apparatus, product, or process disclosed, or represents that its use would not infringe privately owned rights. Reference herein to any specific commercial product, process, or service by trade name, trademark, manufacturer, or otherwise does not necessarily constitute or imply its endorsement, recommendation, or favoring by the United States Government or any agency thereof. The views and spinions of authors expressed herein do not necessarily state or reflect those of the United States Government or any agency thereof.

*Current address: Laboratory for Laser Energetics, 250 E. River Road, Rochester, NY 14623.

* Laboratory for Laser Energetics, University of Rochester, Rochester, NY 14623.

Current address: Imperial College, London SW7 2BZ, ENGLAND.

'Sandia National Laboratories, Div. 1244, Albuquerque, NM 87123.

thission Research Corporation, Los Alamos, NM 87544. 


\section{CONTENTS}

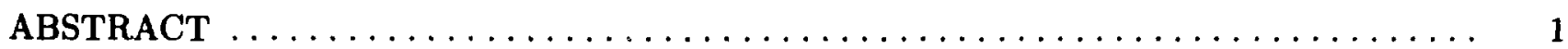

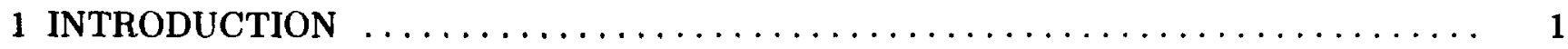

2 APPLICATIONS OF LASER PLASMA-GENERATED X RAYS

AND PARTICLES

2.1 INTRODUCTION TO THE PHYSICAL MECHANISMS INVOLVED

IN THE GENERATION OF X RAYS AND HIGH-ENERGY PARTICLES $\ldots \ldots \ldots . .2$

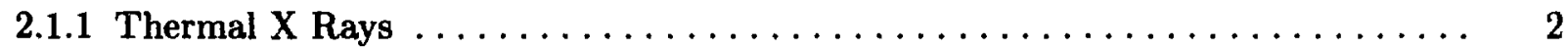

2.1.2 Suprathermal X Rays $\ldots \ldots \ldots \ldots \ldots \ldots \ldots \ldots \ldots \ldots \ldots \ldots \ldots \ldots$

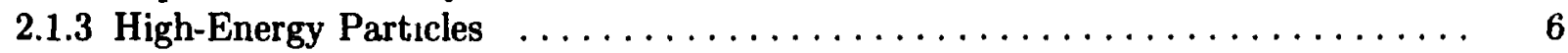

2.2 X-RAY APPLICATIONS $\ldots \ldots \ldots \ldots \ldots \ldots \ldots \ldots \ldots \ldots \ldots \ldots \ldots \ldots \ldots \ldots \ldots$

2.2.1 X-Ray Diffraction from Transiently Strained Crystals $\ldots \ldots \ldots \ldots \ldots \ldots$

2.2.2 Time-Resolved EXAFS $\ldots \ldots \ldots \ldots \ldots \ldots \ldots \ldots \ldots \ldots \ldots \ldots \ldots \ldots \ldots \ldots$

2.2.3 X-Ray Diffraction and Radiography of Biological Samples . . . . . . . . . 15

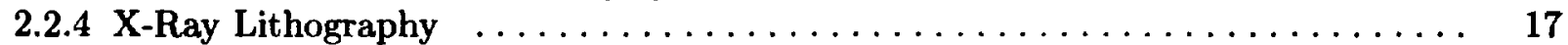

2.3 PARTICLE APPLICATIONS $\ldots \ldots \ldots \ldots \ldots \ldots \ldots \ldots \ldots \ldots \ldots \ldots$

3 LASER-PLASMA ACCELERATION OF PARTICLES $\ldots \ldots \ldots \ldots \ldots \ldots \ldots$

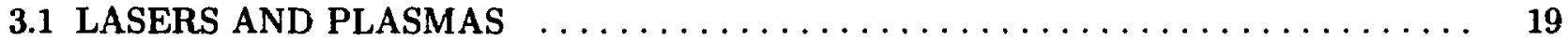

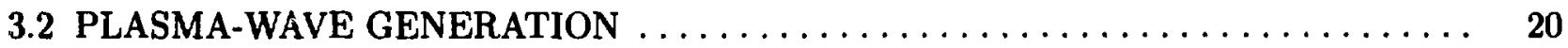

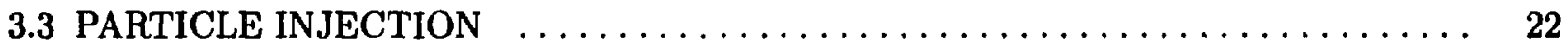

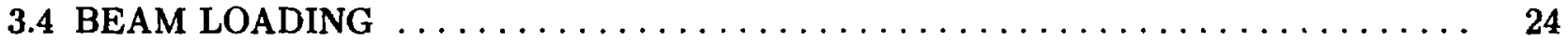

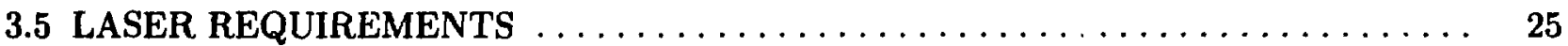

4 LASER PULSED-POWER SWITCHING $\ldots \ldots \ldots \ldots \ldots \ldots \ldots \ldots \ldots \ldots$

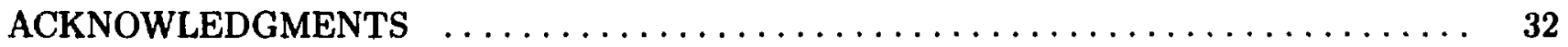

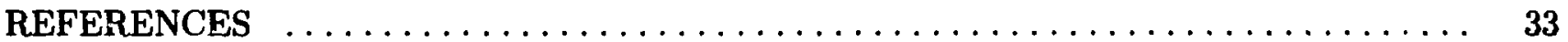




\title{
CURRENT NEW APPLICATIONS OF LASER PLASMAS
}

\author{
by
}

\author{
Allan A. Hauer, David W. Forslund, Colin J. McKinstrie, \\ Justin S. Wark, Philip J. Hargis, Jr., Roy A. Hamil, and Joseph M. Kindel
}

\begin{abstract}
This report describes several new applications of laser-produced plasmas that have arisen in the last few years. Most of the applications have been an outgrowth of the active research in laser/matter interaction inspired by the pursuit of laser fusion. Unusual characteristics of high-intensity laser/matter interaction, such as intense $x$-ray and particle emission, were noticed early in the field and are now being employed in a signiflcant variety of applications outside the fusion field. Applications range from biology to materials science to pulsed-power control and particle accelerators.
\end{abstract}

\section{INTRODUCTION}

The applications of laser-produced plasmas (LPs) have been a wide-ranging field of investigation for more than two decades. In this report, we will review several applications that (with one exception) have been active topics of investigation only for the last few years.

We deal first with the application of lasergenerated particles and $x$ rays. This topic is largely an outgrowth of laser fusion research and primarily employs the higher flux regime with irradiances $>10^{13} \mathrm{~W} / \mathrm{cm}^{2}$. This topic demonstrates a very satisfying diversity of practical applications ranging from biology to materials science, as well as plasma and atomic physics, that has been spawned by basic research in laser/matter interaction.

Next, we address the subject of LP particle acceleration. It has been recognized for many years that extraordinary plasma conditions, such as very high electric fields, are produced at high laser irradiances $\left(>10^{15} \mathrm{~W} / \mathrm{cm}^{2}\right)$. More recently, it has been suggested that these conditions could be used as the key element in a new type of highenergy particle accelerator. The field gradients and other parameters that may be available with these techniques are such that a whole new area of accelerator technology might be opened up.

Finally, we discuss laser switching and controlling of pulsed power. 
2 APPLICATIONS UF LASER

PLASMA-GENERATED X RAYS AND PARTICLES

\subsection{INTRODUCTION TO THE PHYSICAL MECHANISMS INVOLVED IN THE GENERATION OF X RAYS AND HIGH-ENERGY PARTICLES}

The LP can be an efficient, bright, and versatile source of $x$ rays. In some situations as much as $70 \%$ of the absorbed laser light can be re-emitted as $x$ rays. In order to have reasonably efficient radiation in the $x$-ray regime, plasma temperatures in the range of hundreds of electron volts or greater must be generated. In order to reach such conditions, laser irradiances $>10^{12} \mathrm{~W} / \mathrm{cm}^{2}$ must be achieved. With variations in irradiance and wivelength that are allowed by present laser technology, $x$-ray phcton energies can range across the spectrum up to several hundred keV. The $x$-ray emission also has very interesting temporal characteristics. In many cases, the temporal profile follows the envelope of the laser itself. This in turn implies pulse durations ranging from tens of picoseconds (or less) to tens of nanoseconds. The LP can thus be a very high peak-power $x$-ray source. Controlability is another very valuable characteristic of LP x-ray sources. Because laserinitiated electronic and pulsed-power switching (see Section 4) has become highly developed, the $x$-ray source can be accurately synchronized with respect to a wide variety of instruments (e.g., ultrafast optical and x-ray streak cameras and shutters). In addition, the laser itself is often used to stimulate an event (such as a material phase transition), which can then be probed with an x-ray burst. The spaitial distribution of $x$-ray emission can be varied from a point source (as small as $10 \mu \mathrm{m}$ ) to distributed emission (as large as several millimeters) by simply varying the laser focal conditions.

The physica! mechanisms responsible for kilovolt plasma formation and subsequent $x$-ray emission show a marked variation with laser wavelength. Shorter wavelengths $(\lambda<1 \mu \mathrm{m})$ are more efficient for producing quasi-thermal plasma emission (Yaakobi et al., 1981). Longer-wavelength $(\lambda>10 \mu \mathrm{m})$ laser interactions have the additional feature of producing large numbers of suprathermal electrons that in turn produce hard $x$-ray line radiation and bremsstrahlung (Hauer et al., 1984).

Equally remarkable is the particle emission from LPs. The thermal portion of LPs produces an ion blowoff that is very valuable for diagnostics but is less interesting from an application point of view. The aforementioned suprathermal-electron generation is also the driving source for the production of very-high-energy ions. This is usually referred to in the literature as "fast ion" generation. Both the particle energy and efficiency of generation are enhanced by going to longerwavelength laser irradiation, and a $\mathrm{CO}_{2}$ laser is thus the best choice for such work.

It is useful when we consider the mechanisms for LP generation of $x$ rays and particles to refer to the spatial structure of the interaction, as is illustrated in Fig. 1. Laser energy is absorbed, and a portion of it is conducted to a region near the ablation surface where the strongest $\mathrm{x}$-ray emission (>1 keV) occurs. The efficiency of emission is particularly dependent on the incident laser irradiance and wavelength.

Typical density and temperature profiles are shown in Fig. 2. At higher intensities, the profiles tend to steepen, and the regions of laser deposition and maximum $x$-ray emission tend to be of smaller spatial extent.

Both particle and x-ray generation mechanisms can be divided into two broad categories: thermal and suprathermal. The absorbed laser energy can be channeled into either thermal electrons, which produce thermal plasma heating, or suprathermal electrons, which tend to escape or deposit deeply in cold material. We will divide the discussion into two sections dealing with thermal and suprathermal $\mathrm{x}$-ray generation.

2.1.1 Thermal $X$ Rays. In assessing the characteristics of the production of thermal $x$-ray photons of any particular energy, we must consider the following parameter variations:

(a) Laser absorption (for $\lambda<1 \mu \mathrm{m}$ ) increases with decreasing $\lambda$. For $\mathrm{CO}_{2}$ laser irradiation $(\lambda=10.6 \mu \mathrm{m})$ there is a special dependence. 


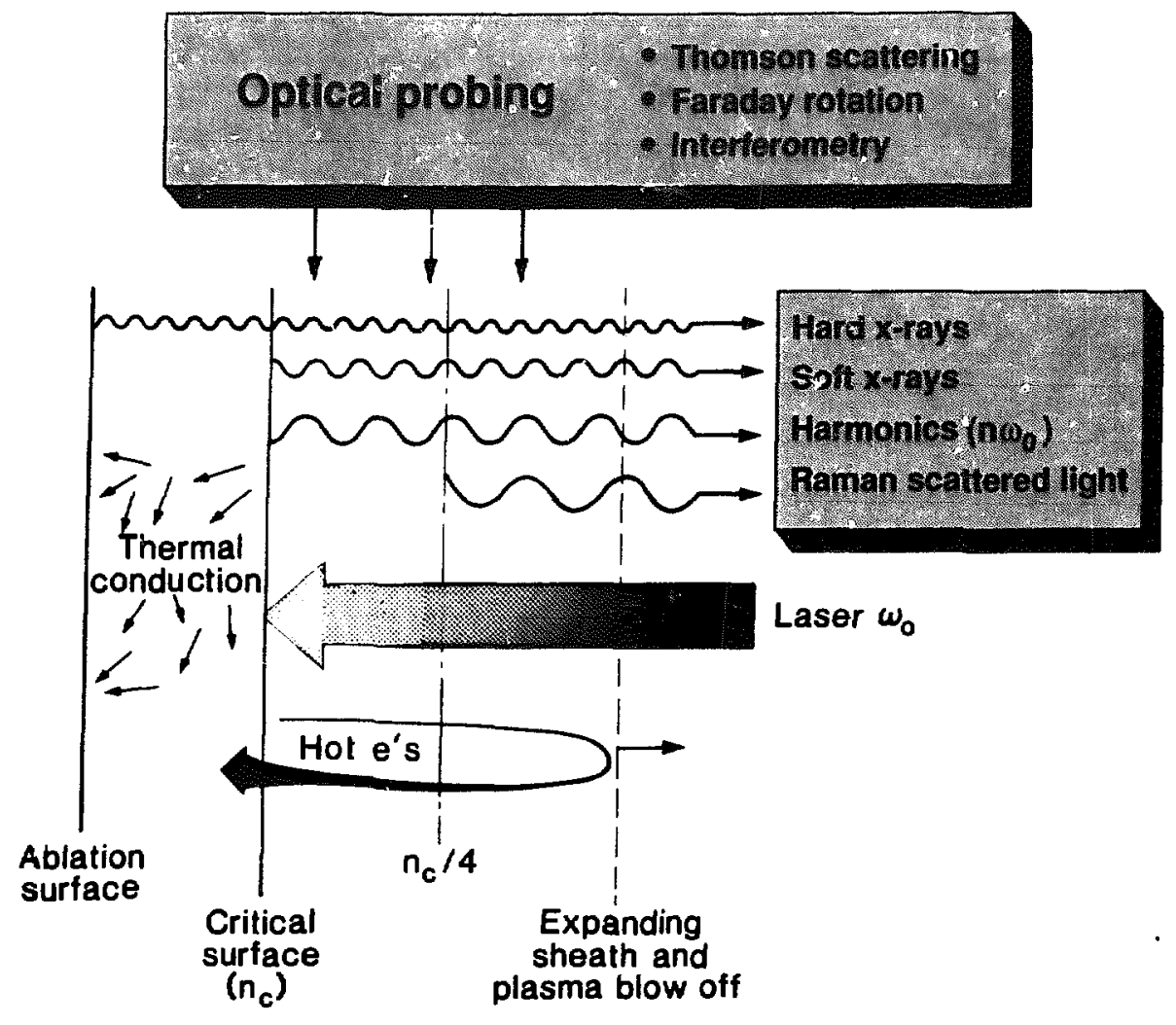

Fig. 1. Laser plasma interaction-absorption/tran'port phase.

Typical experimental data on laser absorption as a function of wavelength and irradiance are given in Fig. 3.

(b) The fraction of energy channeled into hot electrons increases roughly linearly with the parameter $I \lambda^{2}$ (where $I$ is irradiance).

(c) For a given laser wavelength, the intensity must be increased to reach higher $x$-ray photon energies. This is because the rate of energy delivery must be increased to reach higher plasma temperatures and thus higher ionization states.

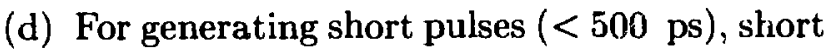
wavelengths should be more efficient because the higher uitical density should lead to a higher collision rate and more rapid achievement of higher ionization states. Several studies of these variations have been made
(Yaakobi et al., 1981; Matthews et al., 1983; Phillion and Hailey, 1986), but parameter studies for all the regimes of interest in applications have not yet been completed. In Table 1 we try to give a rough indication of the range of parameters that are reasonably agreed on in the literature. We define the following efficiency parameter;

$\eta=$ x-ray energy in a particular photon energy band (which is noted) into $4 \pi$ /incident laser energy.

In many applications in materials science and biology, a pulse width of 1-10 ns is adequate for the study of transient phenomena. This pulse width must be balanced against the need to deliver energy fast enough (high laser power) so that high ionization states can be reached and thus relatively-short-wavelength $\mathrm{x}$-ray radiation 
(a)

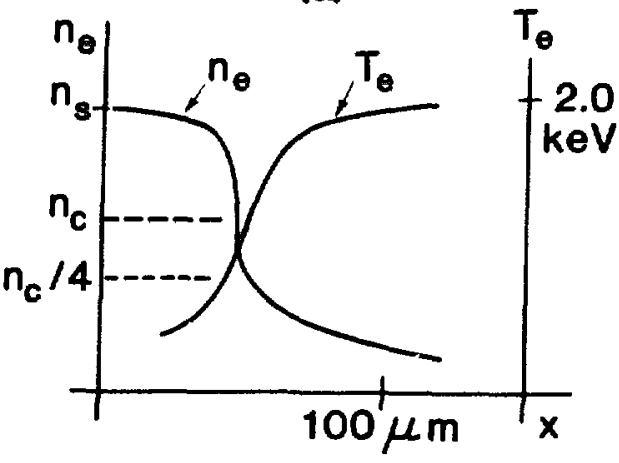

(b)

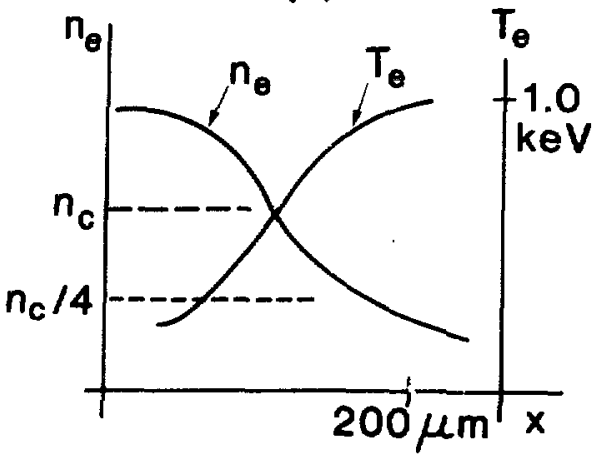

Fig. 2. Typical density and temperature profiles for (a) high- and (b) low-intensity irradiation. $n_{c}$ is the critical density, and $n$, is solid density.

generated (before cooling by thermal conduction, hydrodynamic expansion, and radiation occurs). Radiography of dense plasmas often requires bursts of $100 \mathrm{ps}$ or shorter. This in turn requires the use of shorter wavelength laser irradiation of the $\mathrm{x}$-ray source.

2.1.2 Suprathermal $X$ Rays. The generation of suprathermal $x$ rays is a very different phenomena from thermal emission (Priedhorsky et al., 1981; Burnett et al., 1984; Hauer et al., 1984; Forslund and Goldstone, 1985). In essence, the process amounts to the use of the absorbed laser energy to produce an electron beam, which

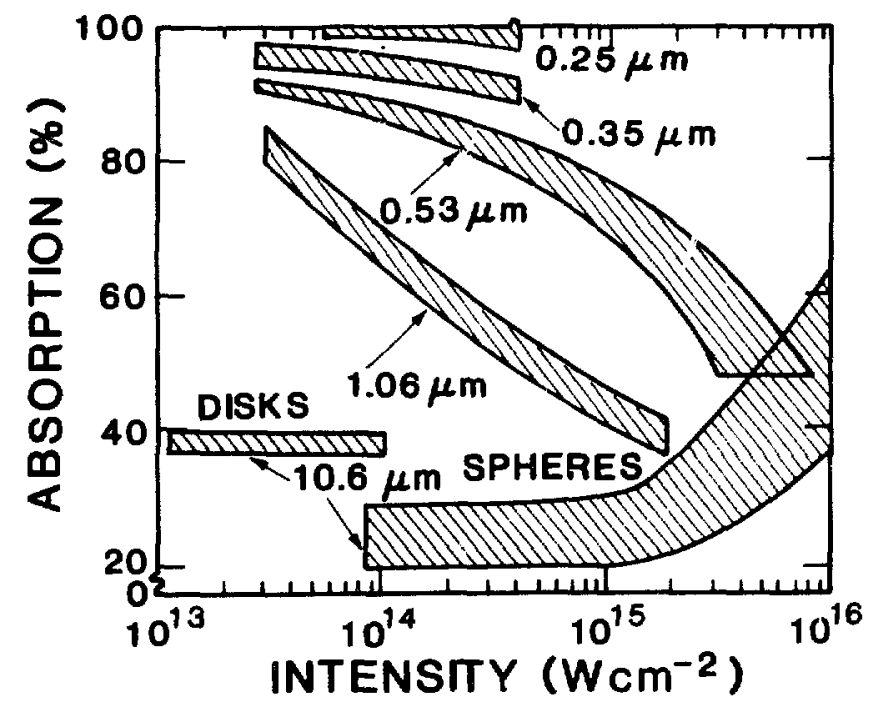

Fig. 3. Laser absorption as a function of wavelength and irradiance. in turn has many similarities to a classical $\mathrm{x}$ ray tube. Referring once again to Fig. 1, we see that suprathermal electrons are usually generated in the region from somewhat below $n_{c} / 4$ to $n_{c}$ (where $n_{c}$ is the critical density). The fraction of absorbed laser energy that goes into suprathermal ("hot") electrons is proportional to the parameter I $\lambda^{2}$. For more precise information on the scaling laws, the reader should consult the references, but with respect to applications one can assume that for $\mathrm{CO}_{2}$. irradiation $(\lambda=10.6 \mu \mathrm{m})$ essentially all of the absorbed energy goes into hot electrons.

A simplified picture of hot-electron generation is adequate to understand the bounds on highenergy $x$-ray generation. Most of the hot electrons are generated near the critical surface. This hot electron energy is resident in a Maxwellian distribution with a temperature designated $\mathrm{T}_{H O T}$. The dependence of $T_{H O T}$ on laser wavelength and laser intensity over a wide parameter range can be very complicated. With respect to applications, however, we restrict our view to the range $10^{15}-$ $10^{17} \mathrm{~W} / \mathrm{cm}^{2}$ and $\mathrm{CO}_{2}$ laser irradiation. In this regime, we can use the following relation:

$$
T_{H O T} \sim(14.8) I^{0.53} \text {, }
$$

where $I$ is in units of $10^{14} \mathrm{~W} / \mathrm{cm}^{2}$ and $T_{H} O T$ in $\mathrm{keV}$. Outward-moving electrons create a charge imbalance, and electrostatic potentials of hundreds of kilovolts can build up. This potential soon forms a sheath of positive ions, which tends to reflect some of the subsequent electron emission. The hot electrons lose some of their energy 
Table 1. Characteristics of Thermal X-Ray Emission from Laser Plasmas

\begin{tabular}{|c|c|c|}
\hline $\begin{array}{l}\text { X-Ray } \\
\text { Emission } \\
\text { Parameters }\end{array}$ & $\begin{array}{l}\text { Characteristics } \\
\text { of X-ray Generation } \\
\text { (for laser wavelength } \lambda \\
\text { and intensity I) }\end{array}$ & Applications \\
\hline $\begin{array}{l}\text { Broadband } \\
\text { subkilovolt } \\
\Delta \nu \sim 0.05-1.0 \mathrm{keV}\end{array}$ & $\begin{array}{l}\text { - for given I considerable } \\
\text { increase of } \eta \text { with } \\
\text { decreasing } \lambda \\
\text { - peak efficiencies } \\
\lambda=0.35 \mu \mathrm{m} \text { is } \eta \sim 0.55 \\
\text { @ I } \sim 10^{14} \mathrm{~W} / \mathrm{cm}^{2}\end{array}$ & $\begin{array}{l}\text { - photo } \\
\text { lithography } \\
\text { - surface } \\
\text { studies }\end{array}$ \\
\hline $\begin{array}{l}\text { Thermal line } \\
\text { radiation } \\
h \nu \sim 2 \mathrm{keV}\end{array}$ & $\begin{array}{l}\lambda=0.35 \mu \mathrm{m}, \eta \sim 0.02 \\
@ \mathrm{I}=5 \times 10^{14} \mathrm{~W} / \mathrm{cm}^{2}\end{array}$ & $\begin{array}{l}\text { - x-ray radio- } \\
\text { graphy (e.g., } \\
\text { biology) }\end{array}$ \\
\hline $\begin{array}{l}\text { (He-like } \\
\text { emission for } \\
\text { elements like } \\
\text { Al) } \\
\Delta \nu \sim 10 \mathrm{eV}\end{array}$ & $\begin{array}{l}-\lambda=1.06 \mu \mathrm{m}, \eta \sim 0.006 \\
@ \mathrm{I}=5 \times 10^{14} \mathrm{~W} / \mathrm{cm}^{2} \\
\\
\text { - recombination rates } \\
\text { generally cause x-ray } \\
\text { pulse }>\text { laser pulse } \\
\text { (for } \tau_{L} \leq 100 \mathrm{ps} \text { ) }\end{array}$ & $\begin{array}{l}\text { - transient } \\
\text { diffraction } \\
\text { from biological } \\
\text { structures }\end{array}$ \\
\hline $\begin{array}{l}\text { Thermal line } \\
\text { radiation } h \nu \sim \\
5 \mathrm{keV} \text { (He-like } \\
\text { emission for } \\
\text { elements like Ti) }\end{array}$ & $\begin{array}{l}-\lambda=0.35 \mu \mathrm{m}, \eta \sim 0.006 \\
@ \mathrm{I}=5 \times 10^{14} \mathrm{~W} / \mathrm{cm}^{2} \\
-\lambda=1.06 \mu \mathrm{m}, \eta \sim 5 \times 10^{-4} \\
@ \mathrm{I}=5 \times 10^{14} \mathrm{~W} / \mathrm{cm}^{2} \\
\text { x-ray pulse usually } \\
\text { closely follows } \\
\text { laser pulse }\end{array}$ & $\begin{array}{l}\text { - transient } \\
\text { diffraction } \\
\text { in materials } \\
\text { science and } \\
\text { biology }\end{array}$ \\
\hline
\end{tabular}

by interacting with this sheath and this interaction can in turn produce ion acceleration (described in the next section). The bulk of the remaining energy is deposited in the dense portions of the target. The stopping of fast electrons in dense matter is due to ionization and excitation of bound electrons, collective loss to free electrons (Jackson, 1975), and bremsstrahlung radiation. Bremsstrahlung and inner-shell radiation excited in this slowing-down process are potentially valuable in applications. A rough indication of the energy appearing in bremsstrahlung can be obtained through the use of the following relation (Birkhoff, 1958):

$$
\frac{\text { Electron Energy Lost To Bremstrahlung }}{\text { Total Deposited Energy }}=\frac{E Z}{800}
$$

where $\mathrm{E}=$ electron energy in $\mathrm{MeV}$.

The hot electrons also produce inner-shell vacancies that are filled by transitions, which produce line radiation. For the applications presently 
being considered, $\mathrm{K}$-shell radiation (e.g., $\mathrm{K}_{\boldsymbol{\alpha}}, \mathrm{K}_{\beta}$ emission) is the most important.

Detailed measurements have been made of the absolute yields of both bremsstrahlung continuum (Priedhorsky et al., 1981) and inner-shell line radiation. Table 2 gives a review of some parts of these data in the context of potential applications. As can be seen from Table 2, suprathermal $x$-ray generation is quite inefficient (as is an ordinary $x$-ray tube). It is, however, one of the few ways to produce an $x$-ray source that combines all of the following characteristics:

(a) high photon energy up to at least $30 \mathrm{keV}$;

(b) small source size $\lesssim 100 \mu \mathrm{m}$; (c) flexibility in changing source configuration by changing focal conditions; and

(d) intense fast $\tau \leq 1$ ns pulsed bursts

In addition, the technology of $\mathrm{CO}_{2}$ lasers has progressed to the point where compact kilojoule, high-repetition-rate $(>0.01 \mathrm{~Hz})$ systems can be planned.

2.1.3 High-Energy Particles. Hot electrons that are confined to the coronal region by the sheath and self-generated magnetic fields (Forslund and Brackbill, 1982; Forslund and Goldstone, 1985) can impart a significant portion of their energy to ions. In $\mathrm{CO}_{2}$ laser experiments

Table 2. Characteristics of Suprathermal X-Ray Emission from Laser Plasmas

Characteristics

of X-Ray Generation

X-Ray Emission (for laser wavelength $\lambda$

Parameters and intensity I)

Applications

Characteristic

$\lambda=10.6 \mu \mathrm{m}$

line emission

$\eta \sim 4 \times 10^{-5}$

$h \nu \geq 8 \mathrm{keV}$

$@ \mathrm{I} \sim 3 \times 10^{15} \mathrm{~W} / \mathrm{cm}^{2}$

$\Delta \nu \sim 5 \mathrm{eV}$

$\mathrm{x}$-ray pulse.

follows laser

pulse for

$\tau_{L} \sim 1$ n.s.

- Transient diffraction from smail 2-D spacing crystals

- Radiography of very dense plasmas

Bremsstrahlung continuum $10-500 \mathrm{keV}$ (measured) $\lambda=10.6 \mu \mathrm{m}$

@ I $\sim 3 \times 10^{15} \mathrm{~W} / \mathrm{cm}^{2}$

$\eta \sim 10^{-3}$

(for total spectrally integrated Bremsstrahlung)
- Transient powder diffraction

- Absorption spectra from very dense plasmas

- Transient effects with respect to radiation damage in solids 
at high intensity $\left(\sim 10^{16} \mathrm{~W} / \mathrm{cm}^{2}\right)$, as much as $60 \%$ of the absorbed laser energy has been measured in protons with $>100 \mathrm{keV}$ energy (Hauer et al., 1984). A very simple model of the ion acceleration gives some feeling for the mechanisms involved. The hot electrons can be thought of as making multiple bounces off the sheath. On each bounce they give up a fraction $\sim 2 \sqrt{Z / A\left(m_{e} / m_{p}\right)}$ to ions, where $\mathrm{Z} / \mathrm{A}$ is the atomic charge to mass ratio and $m_{e}$ and $m_{p}$ are the electron and proton masses, respectively. As a specific example, in a typical $\mathrm{CO}_{2}$ experiment $\left(\sim 2 \times 10^{15} \mathrm{~W} / \mathrm{cm}^{2}\right.$ with $\mathrm{T}_{\text {H }} \sim 120 \mathrm{keV}$ ) about $40 \%$ of the absorbed energy is observed in fast ions. More detailed calculations indicate (Forslund and Brackbill, 1982) that about eight effective bounces may occur. For a gold shell, $8 \times 2 \sqrt{Z / A\left(n_{e} / m_{p}\right)}=0.36$ or close to the observed fraction.

With respect to applications, the fast ion blowoff has another unique property. The ions tend to be emitted in a fairly narrow cone or jet. The self-generated magnetic fields tend to cause a preferential deposition of hot electrons in high field regions. This in turn produces an electric field

$$
E=-\nabla P / n_{e}
$$

where $P$ is the pressure caused by the deposition of hot electrons having a density $n_{e}$. This accelerating field is much larger than it would be without the magnetic field and extends over a region much larger than the laser spot size. This field tends to channel the blowoff into an ion jet normal to the target surface. In Fig. 4, we show typical data on the angular distribution of fast-ion emission. As can be seen, a significant fraction of the ion energy is contained in a narrow cone angle. Another graphic illustration of jet formation is given in Fig. 5. Here a composite $x$-ray and ion emission image clearly displays the formation of an ion jet.

With respect to the applications discussed here, high-energy protons are the most important. In Table 3, we summarize some of the available experimental information that would be useful in applications and for completeness include information on heavier ions. At higher laser irradiances, a significant flux of particles greater than $1 \mathrm{MeV}$ should be produced at efficiencies at or greater than those shown in Table 3.
Table 3. Summary of the Characteristics of LP Fast Ion Generation $\left(E_{\iota}=\right.$ incident laser energy, $E_{,}=$ion energy)

\begin{tabular}{|c|c|c|}
\hline Type of Ion & $\begin{array}{l}\text { Laser Irradiance } \\
\qquad \mathrm{W} / \mathrm{cm}^{2}\end{array}$ & $\begin{array}{l}\text { Ion Emission } \\
\text { Characteristics }\end{array}$ \\
\hline Protons & $10^{20}$ & $\begin{array}{l}E_{.}>100 \mathrm{keV} \\
\text { Total energy }=0.35 \mathrm{E}_{\iota} \\
\text { Energy in } 10^{\circ} \\
\text { cone }=0.15 \mathrm{E}_{\iota} \\
\mathrm{E}_{\mathrm{i}}>500 \mathrm{eV} \\
\text { Total energy }=0.15 \mathrm{E}_{\iota} \\
\text { Energy in } 10^{\circ} \\
\text { cone }=0.05 \mathrm{E}_{\iota}\end{array}$ \\
\hline Protons & $5 \times 10^{13}$ & $\begin{array}{l}E_{,}>100 \mathrm{keV} \\
\text { Total energy }=0.15 \mathrm{E}_{\downarrow}\end{array}$ \\
\hline $\begin{array}{l}\text { Ta ions } \\
(\bar{Z} \sim 40)\end{array}$ & $10^{16}$ & $\begin{array}{l}\mathrm{E}_{t}>100 \mathrm{MeV} \\
\text { Total energy }=0.15 \mathrm{E}_{\iota}\end{array}$ \\
\hline
\end{tabular}

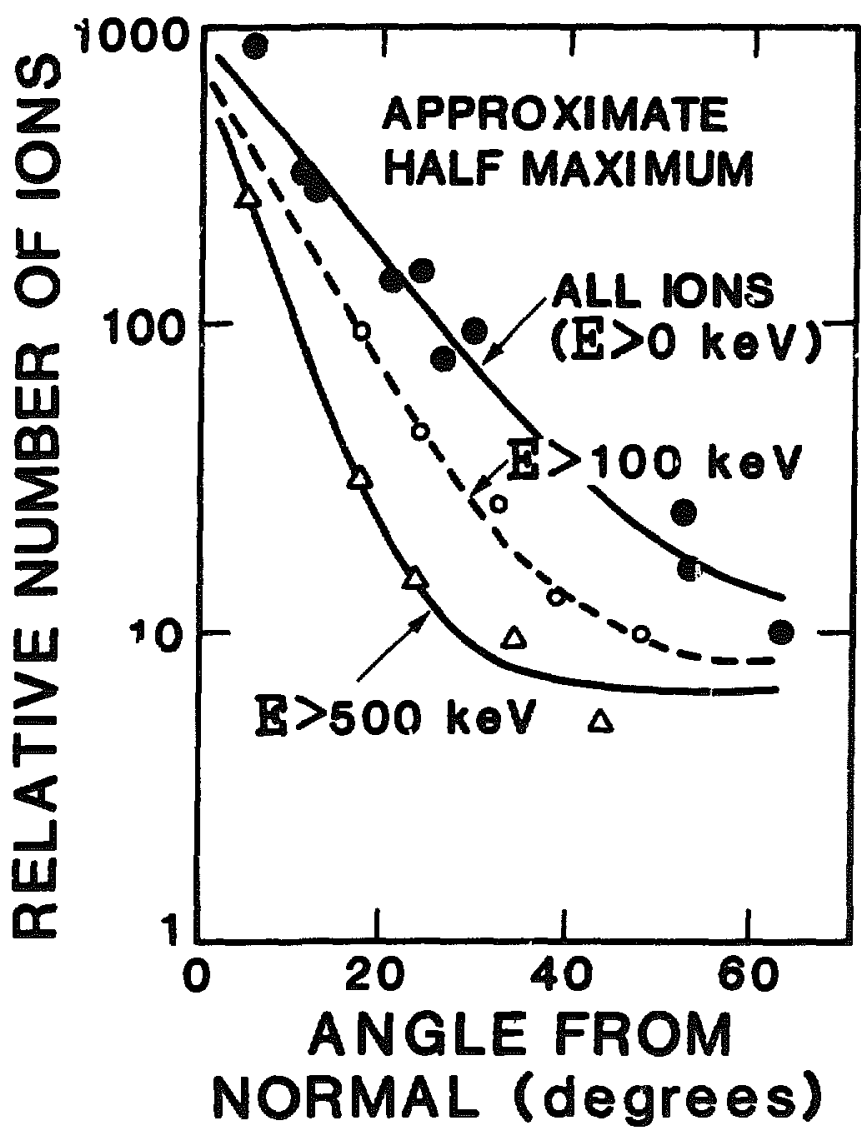

Fig. 4. Angular distribution of fast-ion emision. 


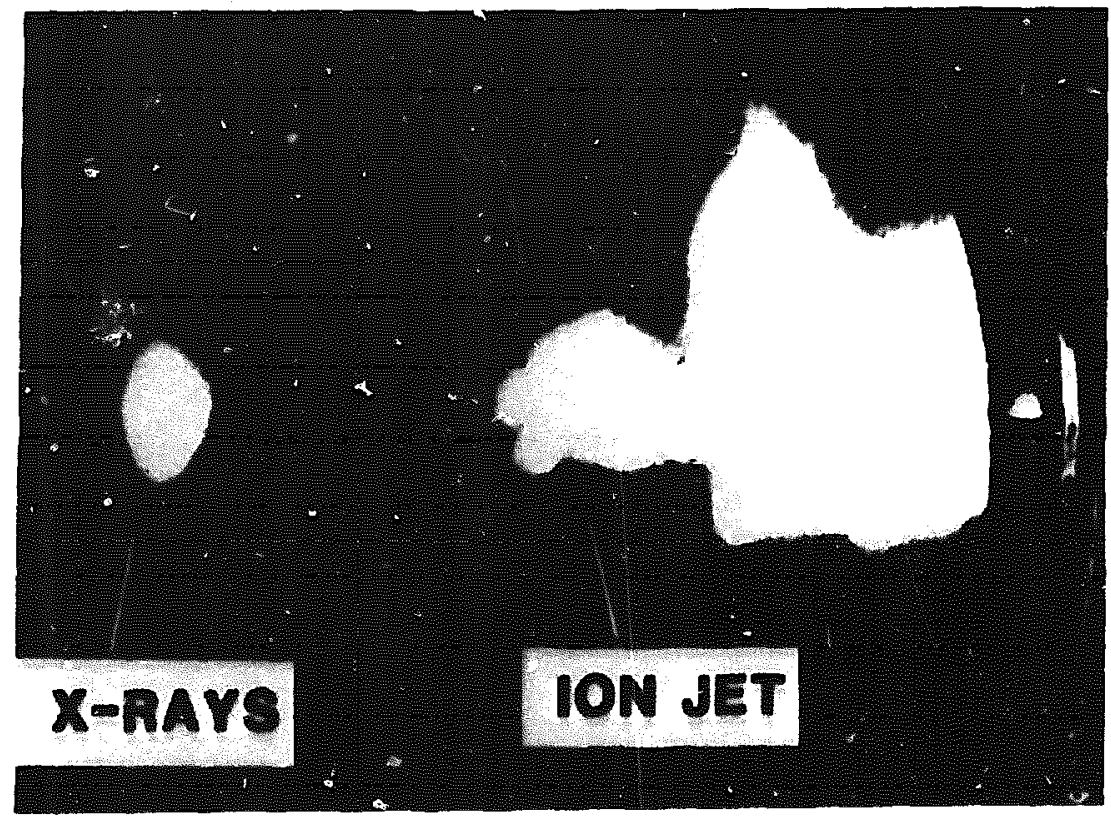

Fig. 5. Composite $x$-ray/ion photograph showing the formation of an ion jet.

\subsection{X-RAY APPLICATIONS}

The unique potential of LP $\mathrm{x}$-ray sources in nonfusion applications has been recognized for some time (Hauer, 1976; Mallozzi, Epstein, and Schwerzel, 1979). Considerable improvement in parameters such as repetition rate and reliability for Nd-glass, $\mathrm{CO}_{2}$, and $\mathrm{KrF}$ lasers has led to a substantial amount of work on nonfusion applications in recent years (Eason et al., 1986; Yaakobi et al., 1986). Most of the applications make use of the fast intense $x$-ray burst that can be produced with LPs. The energy available in a single burst is often adequate for detection and recording. LP $x$-ray sources are thus (in contrast to synchrotron radiation) uniquely suited to the study of nonrepetitive or irreversible events (such as some types of materials phase changes). Laser systems suitable as $x$-ray sources will soon be capable of $>1-\mathrm{Hz}$ repetition rate, and thus LPs will even be competitive on a quasi- $\mathrm{CW}$ basis. X-ray fluence arailable with existing laser systems ranges up to $10^{36} \mathrm{keV} / \mathrm{keV} /$ steradian with photon energies up to $10 \mathrm{keV}$ or greater.

The applications discussed here fall into three broad categories: (a) $x$-ray diffraction from transiently strained crystals, (b) pulsed $x$-ray radiog- raphy and lithography, and (c) absorption spectroscopy.

\subsubsection{X-Ray Diffraction from Transiently} Strained Crystals. The fast burst of $x$ ray emission that is available from LP sources is uniquely suited to transient $x$-ray diffraction (Forster et al., 1984; Hauer, 1976). Using currently available lasers, investigators can record diffraction patterns from a wide variety of crystalline materials on a single shot.

There is currently much interest in the use of pulsed lasers to anneal crystals by transient heating of the crystal surface. A good understanding of the structural dynamics requires a knowledge of the temperature-depth profiles within the crystal as a function of time. One means of probing thes - rofiles is by short-pulse $x$-ray diffraction.

The basic idea is that when the crystal is irradiated by the laser, it heats up; the lattice spacing then increases by an amount determined by the coefficient of thermal expansion. Any $x$-ray radiation incident on the crystal is thus diffracted at a lower angle than that for unheated material. This is easily seen by simple differentiation of Bragg's law: 


$$
\Delta d / d=-\operatorname{Cot} \theta \Delta \theta
$$

where $d$ is the spacing between diffraction planes and $\theta$ is the Bragg angle. In practice, there is a thermal gradient within the crystal, and thus there will be a range of angles at which diffraction takes place up to a maximum angle corresporiding to the hottest part of the crystal, which is assumed to be at the surface of the solid phase. The $x$ rays from a LP allow sucn a Bragg diffraction pattern to be recorded on a single-shot basis with a probe time as low as 100 ps (similar techniques utilizing synchrotron radiation need multiple shots).

Several experiments have been performed in this area using a LP $\mathrm{x}$-ray source (Lunney et al., 1986; Wark, Hauer, and Kilkenny, 1986). The experimental setup of Lunney et al. (1986) is shown in Fig. 6. The silicon (111) crystal is irradiated with $1.05-\mu \mathrm{m}$ light at a fluence of $\sim 0.4$ $\mathbf{J} \mathrm{cm}^{-2}$ in a pulse length of $1 \mathrm{~ns}$ FWHM. After the heating beam, a pulse of $x$ rays is produce. by tightly focusing another laser beam, synchronous and delayed with respect to the first, onto a polyvinyl cloride (PVC) target. At such high irradiances $\left(\sim 10^{16} \mathrm{~W} / \mathrm{cm}^{2}\right)$, He-like chlorine is produced, which has a resonance line at $4.44 \AA$. Bragg diffraction patterns from the heated and unheated portions of the crystal were obtained for various delay times between the heating beam and the $x$-ray probe beam. The spectrum from the heated and unheated parts of the crystal can then be deconvolved to yield the rocking curve (reflectivity as a function of angle around the Bragg angle) as a function of time. The rocking curve of a silicon crystal heated to close to the melting point is two orders of magnitude wider than the natural width. It is possible to extrart the temperaturedepth profiles from these rocking curves using $d y$ namical diffrac ion theory; the technique has been used for several years to obtain strain-depth profiles from crystals strained mechanically, by the introduction of dopants, as well as from induced thermal gradients. We refer the reader to the work of Burgeat and Taupin (1968) and Klar and Rusticelli (1973) for a full explanation of the theory. It suffices to say here that the basic idea is to solve Maxwell's equations to first order within a medium of complex and periodic refractive index (i.e., the crystal). The strain is introduced

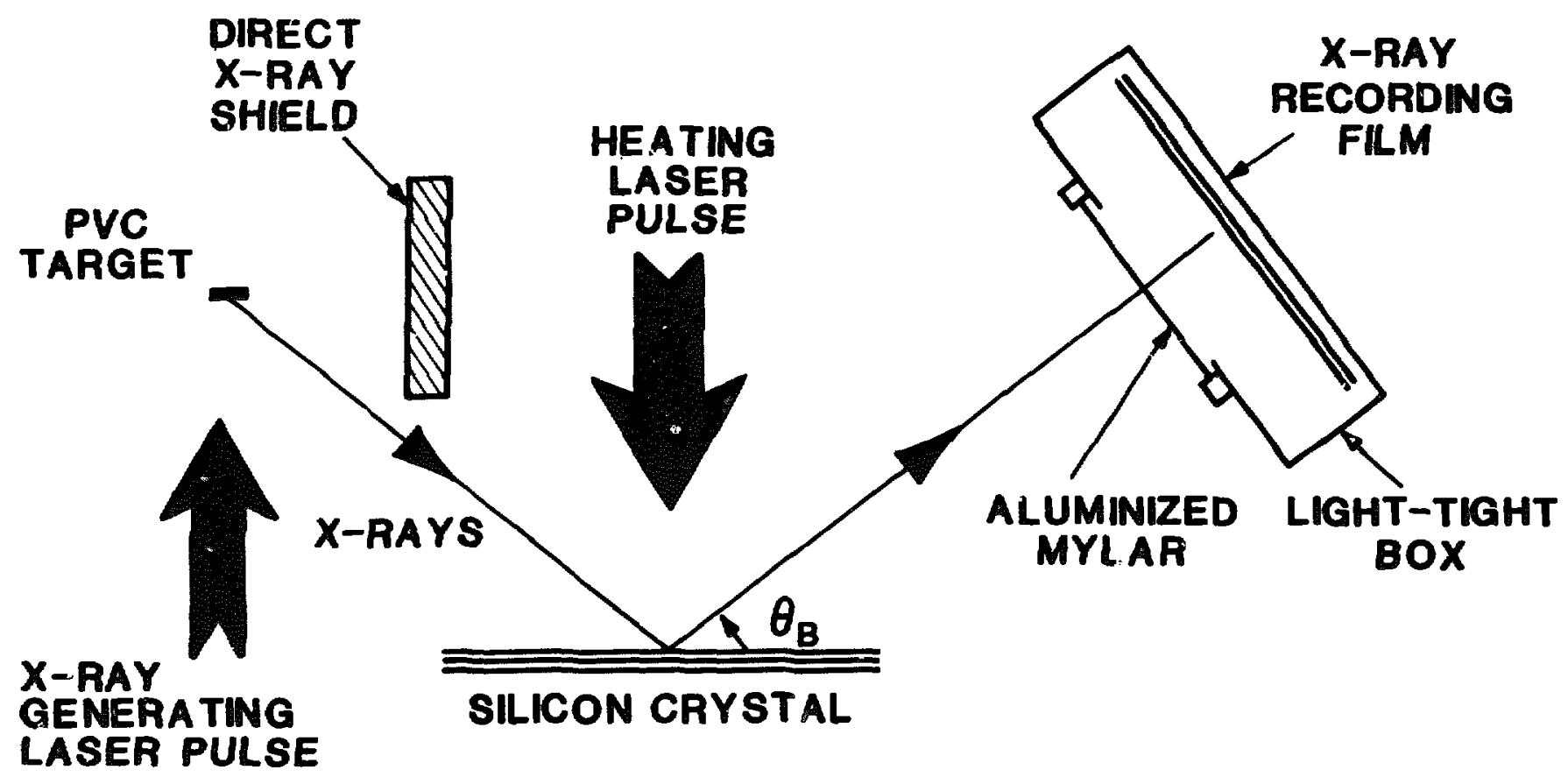

Fig. 6. Experimental configuration used to probe laser-annealed crystals with short LP x-ray bursts (from Lunney et al., 1986; used with permission). 
by a depth-dependent Bragg angle. A monotonic strain-depth profile is guessed and the rocking curve calculated. The calculated rocking curve is compared to that obtained experimentally, and the guessed strain altered accordingly. Thus, the iterations proceed until the experimental and calculated rocking curves agree within some predetermined limits; at this point the strain profile used in the calculations is taken to be the actual profile within the crystal. The temperature-depth profile is obtained from the strain profile using temperature-dependent coefficients of expansion and allowing for the one-dimensional nature of the expansion. The temperature profiles for the experiment described above are shown in Fig. 7. The curves in Fig. 7 were calculated for data from Lunney et al. (1986). Such profiles can be compared to detailed modeling of the laser/crystal interaction using theoretical techniques. The information gained from such comparisons will help to optimize the laser annealing conditions. At higher irradiances, this pulsed diffraction diagnosis may help to reveal important characteristics of the onset of plasma formation (Hauer, 1976).

Transient diffraction studies have also been performed on a subnanosecond time scale (Wark, Hauer, and Kilkenny, 1986). The first motivation in this work was to investigate the possibility of laser heating as a method for $x$-ray switching

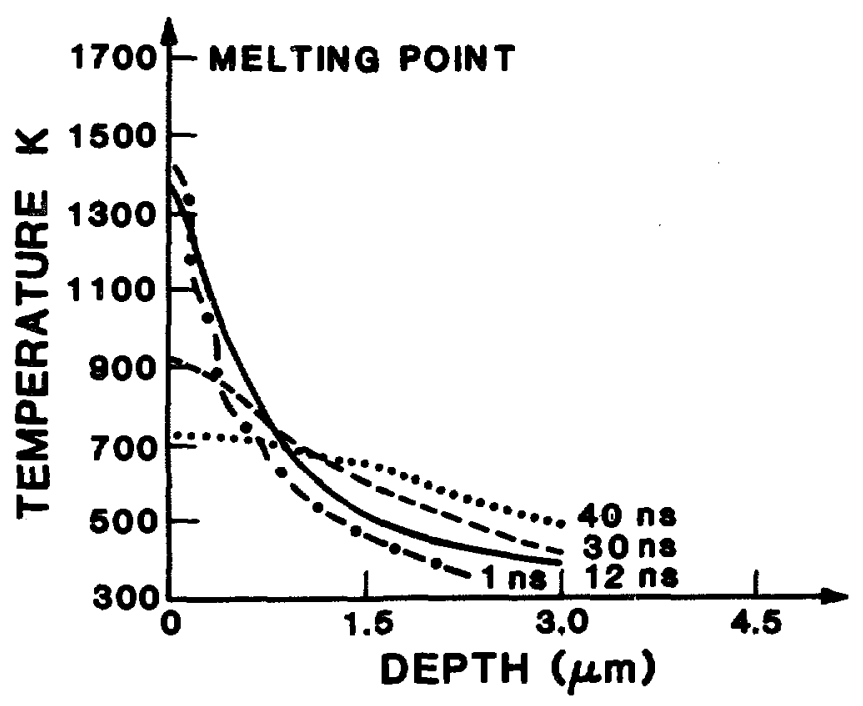

Fig. 7. Temperature-depth profiles within a laserheated cryotal at various probe times. and shuttering. Concepts for doing this are illustrated in Fig. 8. To obtain an "on" switch of line radiation, a beam block is placed in the way from the positive $\Delta \theta$ side, just obscuring the line. Upon irradiation, the rocking curve broadens to negative $\Delta \theta$, allowing radiation to pass through. The switching ratio here is high, between two and three orders of magnitude, but good resolution of the line is lost. An "off" switch may be made utilizing a double-crystal arrangement, as shuwn in Fig. 8. The radiation is double-Bragg reflected off parallel crystals. Upon illumination of the first crystal, the diffracted radiation that now satisfies the Bragg criterion has fallen in intensity because of the reduction in peak reflectivity of the first crystal; i.e., the irtensity falls by about a factor of 20. This arrangement would also be compatible with an extended source of $x$ rays, providing the angular size of the source is small compared with the angle between two diffracted lines. One configuration used for testing these concepts is very similar to that used in Fig. 6, with two important exceptions. Both the heating and $x$-ray source beams were of about 1-ns duration. The time resolution in this case was provided by using a streak camera for detection of the diffracted $x$ rays. The experimental data are shown in Fig. 9. Figure 9(a) shows the temporally resolved spectrum of the radiation diffracted from an unirradiated crystal. The two lines are the resonance and intercombination line of He-like chlorine. The dielectronic satellite lines, which appear as a broad band on the higher-wavelength side of the other two pictures, are not, present here because of slight misalignment of the crystal, causing the satellites to miss the photocathode. Figure 9 (b) shows the diffracted radiation from an illuminated crystal with the peak of the heating beam delayed with respect to the peak of the $x$-ray-producing beam by 500 ps. The lines are seen to broaden and merge together toward the end of the pulse. In Fig. 9(c), there is no delay between the heating and $x$-ray-producing pulse, resulting in broadening early in the pulse. The broadening completely smears out the three original peaks discernible at the start of the pulse. From the angular separation of the peaks (of order 1000 arc-sec), we gain the approximation of the width of the rocking curve to be at least 500 arc-sec. This com- 


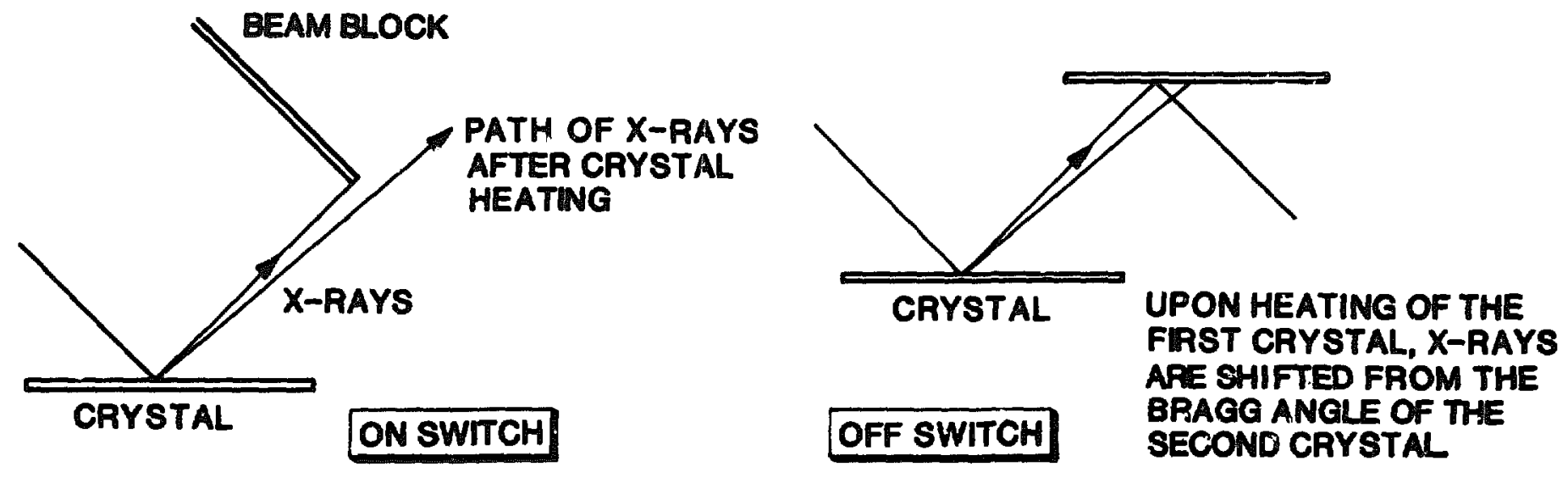

Fig. 8. Concepts for producing x-ray switching and shuttering.
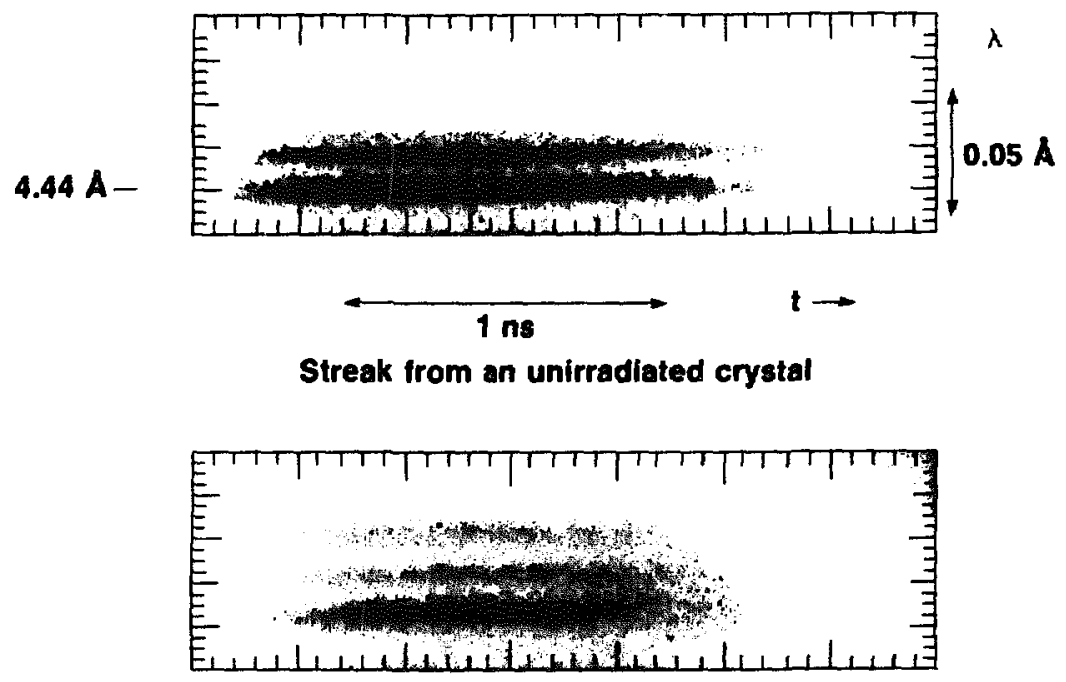

Heating pulse 500 ps after $x$-ray-producing pulse

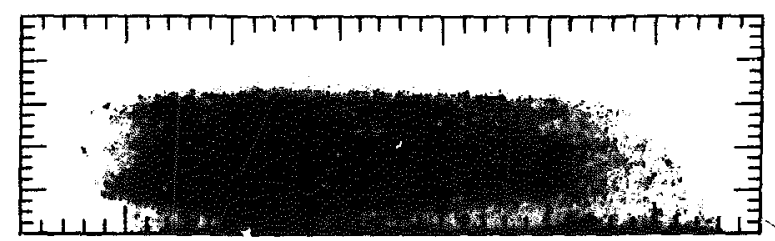

Heating pulse coincident with $x$-ray-producing pulse

Fig. 9. (a) Time-resolved $x$-ray spectrum from a crystal with no heating pulse, (b) heating pulse $500 \mathrm{ps}$ after $x$-ray-producing pulse, and (c) heating and $x$-ray-producing pulses coincident. 
pares with an original width (in the unperturbed crystal) of the order of 50 arc-sec.

In the absence of a double-crystal arrangement, the rocking-curve width of the unirradiated crystal was estimated by placing $x$-ray film in the place of the streak camera (the instrumental broadening of the streak camera was of the order of 250 arc-sec FWHM). From the structure of the dielectronic satellite features in the resultant spectrum, we estimate the rocking-curve width to be of the order of 50 arc-secs. The contribution to the spectral broadening from the width of the $30-\mu \mathrm{m}$ source was of the order of $\mathbf{3 0}$ arc-sec.

A similar theoretical analysis was performed on these data as that described above. From the time-dependent rocking curves and unperturbed crystal parameters, it was found that several hundred arc-seconds away from the Bragg angle the reflectivity changes from an order of $10^{-5}$ to 5 $\times 10^{-2}$ in less than $200 \mathrm{ps}$. The usefulness of such switching properties for diagnostic purposes in fields such as laser fusion may ultimately depend on the maximum obtainable temporal resolution (in this experiment the switching time is less than $200 \mathrm{ps}$ ).

Transient diffraction techniques have also been applied to the study of laser-generated shock waves (Wark et al., 1987). Laser generation of multikilobar shocks has several applications to the study of high explosives (Yang, 1974), highvelocity impacts (Pirri, 1977), the alteration of the mechanical properties of certain alloys and ceramics (Fairand et al., 1972), and solid-state phase changes. For the more conventional shock drivers, the shock-launching region, i.e., the initial layer of the material of interest from which the shock begins, is obscured by the driving mechanism, whether it be a flyer plate, tamper, explosive material, etc. For this reason, well-resolved experimental studies of the shock-launching region have been intractable. Laser-driven shocks do not present this limitation. Some of the most common methods of studying such shock waves include interferometric methods (Sheffield and Fisk, 1983 and 1984) or the use of piezoelectric transducer gauges (Graham, Nelson, and Benedick, 1965). However, these methods yield little information on the dynamics of the nearfront face, where the shock wave is initiated. Probing of the surface by short-pulse $x$-ray diffrac- tion can overcome this problem. The brightness and duration of the $x$-ray pulses from LPs allow unique measurements of the density in the shocked region.

A laser-generated shock is launched into the crystal of interest. At some point during the shock launching, a short (100-ps) pulse of $x$-ray line radiation (produced by a laser beam synchronous and delayed with respect to the shock-generrting beam) is Bragg diffracted off the front surtace of the compressed crystal. The compression of the crystal changes the Bragg condition according to Eq. (4). Thus, the line radiation is diffracted at a higher angle than that for the unperturbed crystal, and this angular shift immediately tells us the change in the interatomic spacing within the shocked region. As there will be a density gradient at the crystal surface, there will be a range of angles at which diffraction takes place, as well as an overall shift. Thus, information about the density gradient, as well as peak density, can be obtained, analogous to the temperature gradient information in the annealing experiment. Indeed, it should be possible to extract a density profile from the data contained in the diffracted pulse.

An experiment of this sort was performed on the JANUS laser system at the Lawrence Livermore National Laboratory. The shocked targets consisted of $250-\mu \mathrm{m}$-thick silicon (111) wafers $5 \mathrm{~cm}$ in diameter, the surface of which had been coated with $1000 \AA$ of aluminum and then $25 \mu \mathrm{m}$ of plastic $(\mathrm{CH})$. The motivation for such a target design is explained below. Half of the target was irradiated with the 1-ns pulse of $1.06-\mu \mathrm{m}$ laser light at a fluence varying from 0.8 to $8 \mathrm{~J} \mathrm{~cm}^{-2}$ with a beam diameter on target of $\sim 4 \mathrm{~cm}$. A beam block prevented irradiation of the other half of the target; diffraction from this unshocked region gave a reference point from which to measure the changes in Bragg angle. After the shock had been launched in the silicon crystal, a second laser beam containing $\sim 10 \mathrm{~J}$ of $0.53-\mu \mathrm{m}$ light in $100 \mathrm{ps}$, which was synchronous with known and variable delay with respect to the shock-launching beam, was focused to a $40-\mu$ m-diameter spot on a calcium-containing target. The He-like calcium lines thus produced were Bragg diffracted off the silicon and recorded on x-ray film.

The rationale benind the target design was that the absorption length of silicon at room temperature to $1.06-\mu \mathrm{m}$ light is several $\mathrm{mm}$; previous ex- 
periments have shown that, under similar irradiance conditions to those used here, uncoaced silicon is simply heated by the laser light, rather than shocked. The aluminum coating, therefore, acts as an absorber for the incident radiation, driving the shock into the material. Overcoating the aluminum with plastic transparent to $1.06-\mu \mathrm{m}$ light causes the expanding aluminum plasma to be inertially confined between the silicon and plastic, which in turn increases the strength of the shock launched into the silicon. Such overcoating techniques have been used to enhance shock pressures in the range of tens of kilobars for several years (Anderholm, 1970).

A typical diffraction pattern is shown in Fig. 10. The three lines in the unshocked region are the resonance line $(3.179 \AA)$, intercombination line
(3.196 $\AA$ ), and a group of unresolved dielectronic satellites of He-like calcium (Feldman et al., 1974). It can be clearly seen that in the shocked region of the crystal the diffracted radiation is broadened and shifted to greater angle, corresponding to a reduction in the lattice spacing. Thus, a simple measurement of the maximum angular shift gives us a direct measurement of the peak density, and knowledge of the broadening yields information on the distribution of lattice spacings within the probed region.

The probe depth of the $x$ rays in such highly strained crystals is determined by the photoelectric absorption coefficient rather than the extinction length. This is because $x$ rays incident at a particular angle only diffract off that thin region of the crystal at which the lattice spacing is such

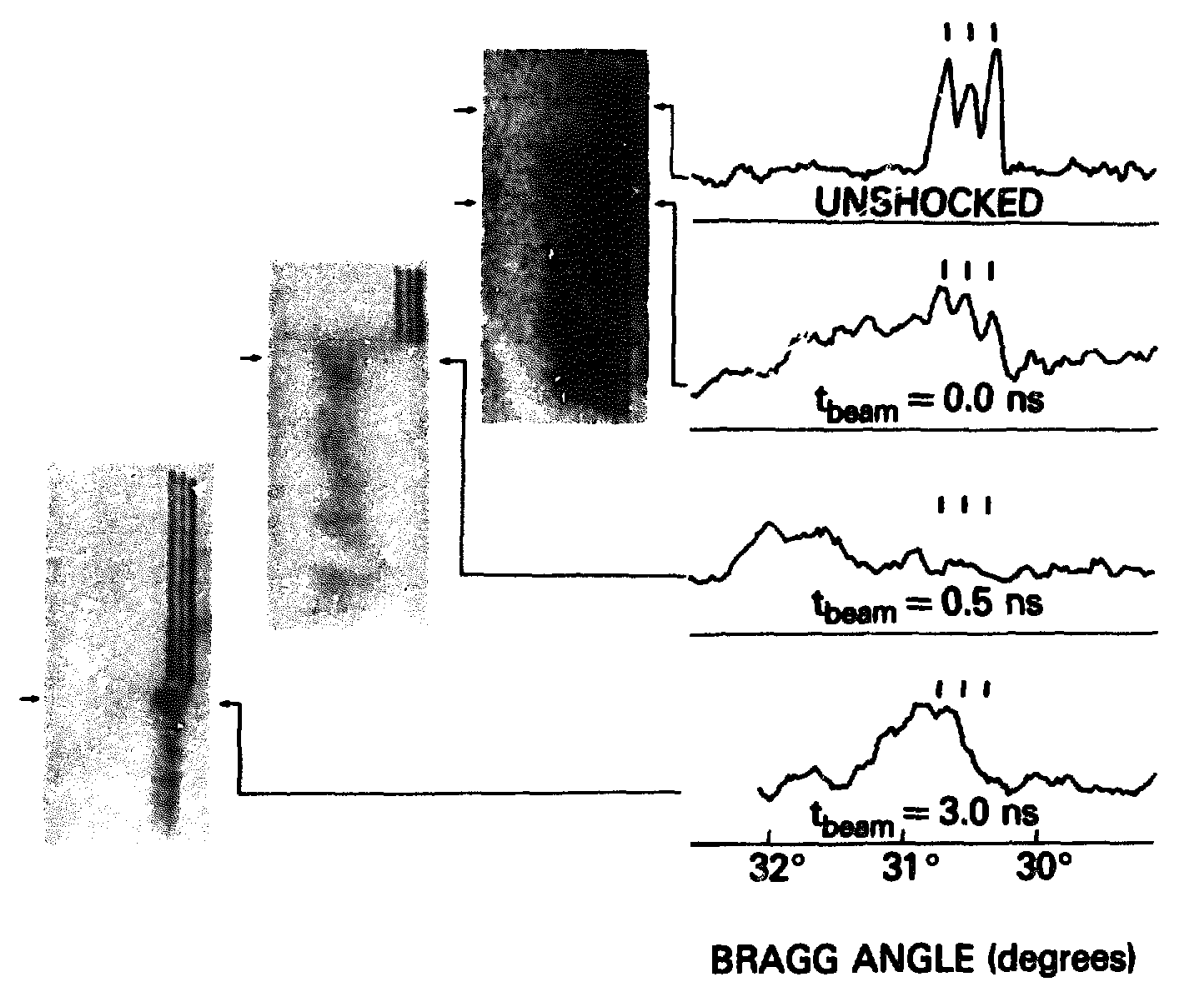

(a)

(b)

Fig. 10. (a) X-ray line spectra diffracted from silicon shocked at an incident laser energy density of $4 \pm 0.3$ $\mathrm{J} \mathrm{\textrm {cm } ^ { - 2 }}$ (an irradiance of $4 \times 10^{9} \mathrm{~W} / \mathrm{cm}^{2}$ ) are shown adjacent to reference lines simultaneously diffracted by the unstrained silicon lattice from a series of beam delays. Each photograph represents a separate shot and beam delay. (b) Densitometer scans, taken at indicated locations through the spectra in (a), are shown for a typical unshocked spectrum and shocked spectra at $0.0-n s, 0.5-n s$, and 3.0 -ns beam delays. 
that the Bragg condition is satisfied; the rest of the crystal up until that point simply acts as an $x$-ray filter. The calcium He-like lines have a $1 / e$ depth in silicon of $\sim 10 \mu \mathrm{m}$. Taking into account the angle of incidence and the dynamic range as determined by the $x$-ray film, a maximum probe depth of between 4 and $6 \mu \mathrm{m}$ is estimated.

Diffraction measurements, such as that shown in Fig. 10, were made for a variety of levels of irradiance and delay times, with zero delay being defined as the point where the peaks of the short and long pulse were coincident. Thus a measurement of the peak density is a function of time for those shots where the average fluence was $4 \mathrm{~J} \mathrm{~cm}^{-2}$. A maximum change of the lattice spacing of $3.5 \%$ was observed, and the dats are consistent with a pressure pulse with a FWHM of 1 ns.

It can be seen that at 0 -ns delay, the unperturbed part of the crystal can still be probed, i.e., we still observe diffraction at the original Bragg angle as well as diffraction from a range of lattice spacings up to a compression of $2.2 \%$. The obvious physical explanation for this is that at this early time the foot of the shock wave has not yet penetrated past the maximum probe depth, whereas for all data collected after this probe time, when the shock has proceeded further into the material, we no longer observe diffraction from the unperturbed crystal. It is interesting to note that, even 5 ns after the peak of the shockproducing pulse, the surface of the crystal is still slightly under compression, i.e., the rarefaction wave was not observed. This is due to the fact that the aluminum plasma is tamped between the silicon and plastic overcoat; this observation is in agreement with hydrocode predictions.

These recent experiments open up unique possibilities for the study of transient phenomena within matter. Some of the more exciting prospects include the time-resolved study of phase changes and the properties of materials within the elastic-plastic transition, as well as the dynamics of materials in a high-radiation flux environment.

2.2.2 Time-Resolved EXAFS. X-ray diffraction is not the only way to obtain information about the interatomic spacings within a material; another method is to observe the EXAFS (extended x-ray absorption fine structure).
In this technique, the $\mathrm{x}$-ray absorption coefficient of the material of interest is studied as a function of energy just above the $K, L$, or $M$ absorption edge. As the name suggests, on the high-energy side of the edge, fine structure in the absorption coefficient exists, and this structure can yield information about the local environment surrounding an atom. In particular, measurements of the bond lengths and coordination numbers can be made. The theoretical interpretation of this structure and the means by which physical information can be extracted were first put forward by Sayers, Stern, and Lytle (1971). A few years later EXAFS spectra were recorded using a synchrotron as the x-ray source (Kincaid and Eisenberger, 1975). Since that time, interest in the technique has expanded rapidly, and EXAFS spectra are now regularly recorded at many synchrotron facilities.

The first spectra using a LP as the $x$-ray source were recorded by Mallozzi et al. (1979), and more recently spectra of higher quality have been recorded by Eason et al. (1984) and Bradley (1985). These initial measurements were made with a static sample using a 1-ns probe pulse. The short recording time afforded by LPs allows measurements to be made on samples that would normally suffer radiation damage during repetitive synchrotron radiation exposure. However, the real advantage of the LP source is the availability of high temporal resolution, which allows bond lengths and coordination numbers to be measured in materials undergoing transient changes on a subnanosecond time scale.

In the first EXAFS measurement with such time resolution, a 1-ns pulse of $x$ rays was produced by tightly focusing $\sim 40 \mathrm{~J}$ of $0.53-\mu \mathrm{m}$ light onto a bismuth target. At such irradiances, the $\mathrm{x}$ ray spectrum of highly ionized bismuth includes a broad band of emission around the K-edge of aluminum at $1.5 \mathrm{keV}$. The $\mathrm{x}$ rays passed through a $3-\mu \mathrm{m}$-thick aluminum foil and were diffracted off a flat thallium acid phthalate (TIAP, $2 \mathrm{~d}=25.9 \AA$ ) crystal onto an x-ray streak camera. A sequence. of such shots was taken, with the $x$-ray source moved closer to the aluminum foil on each successive shot. Thus the foil was transiently heated as well as probed by the $x$ rays. Typical data from this experiment are shown in Fig. 11, for which the foil was $\sim 30 \mathrm{~mm}$ away from the $\mathrm{x}$-ray source. 


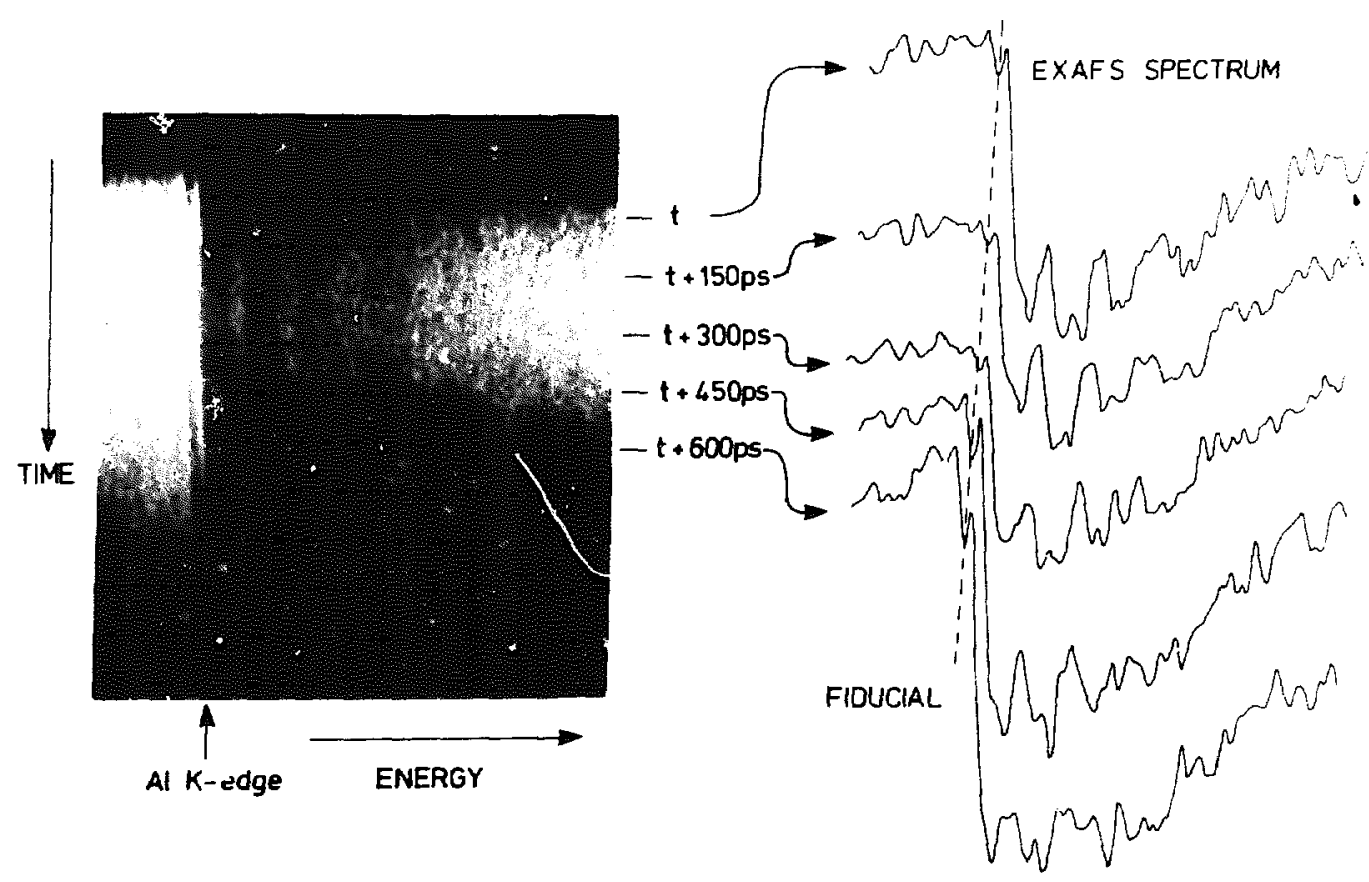

Fig. 11. Typical streaked EXAFS spectrum recorded using a 1-ns $x$-ray pulse (from Eason et al., 1984; used with permission).

Further shots with the foil $3 \mathrm{~mm}$ and $4 \mathrm{~mm}$ from the $x$-ray source show evidence of gross changes in the fine structure during the pulse. At these distances, calculations show that the foil should be melted by the $\mathrm{x}$-ray flux. A preliminary analysis of this data by Bradley (1985) has shown clear evidence of an increase in the interatomic spacing during the 1-ns pulse, with an expansion of $\sim 20 \%$ by the end of the pulse.

The use of EXAFS to explore subnanosecond phenomena has been proven. The two techniques of Bragg diffraction and EXAFS complement each other well. Almost any structural change that can be synchronized to the laser can be probed by one or both of these techniques, and thus we can look forward to a significant increase in our understanding of transient phenomena over the next few years.

\subsubsection{X-Ray Diffraction and Radiography} of Biological Samples. The study of living cells is of obvious fundamental importance in medical and biological research. Although much can be learned using conventional microscopy in the optical region, a basic limitation is placed on the maximum spatial resolution by the wavelength of the light used. The resolution can obviously be improved by probing with shorter wavelengths, i.e., electron microscopy or soft-xray contact microscopy. However, in electron microscopy, the sample must be dried and coated before observations can be made; the cell is thus killed and the sample may appear vastly different than its living counterpart. Similar problems occur with $\mathrm{x}$-ray microscopy when a conventional $x$-ray source is used. A contact print of the sample is taken by irradiating it with $\mathrm{x}$ rays over a period of several hours. During this time, the cell suffers severe radiation damage and is killed during the course of the exposure. If a 1-ns burst of LP $\mathrm{x}$ rays is used as the source, this problem is overcome; the radiation damage occurs on a microsecond time scale; thus a print of the living cell is obtained before cell death occurs.

The use of LP $\mathrm{x}$-ray sources in this field has been applied by Michette, Eason, Cheng, and coworkers to study a host of biological and botanical specimens. We refer the reader to their excellent works (Michette et al., 1986; Eason et al., 1986) for details of the particular results; only 
the general technique will be described here. The living cells exist within the necessary nutrients under atmospheric pressure. $A$ thin $\mathrm{Si}_{3} \mathrm{~N}_{4}$ window, transparent to the probing $x$-rays, separates them from the surrounding vacuum. The detector in these experiments is a photoresist where the image is formed by radiation damage in a plastic material. The radiation damage centers then cause selective etching when the plastic is exposed to an acid. The cells are in intimate contact with a copolymer $x$-ray resist. The LP soft $x$ raye pass through the cells and produce a latent image within the resist, corresponding to a two-dimensional image of the line-integrated mass-absorption coefficient of the sample. Subsequent etching yields an image of the cell as a relief pattern within the resist.

To obtain high-contrast images of the specimens, the $x$-ray wavelength is chosen to be within the carbon and oxygen $K$ absorption edges (between 44 and $23 \AA$ ). Thus, the water within the samples is relatively transparent to the $x$ rays, whereas the carbon and nitrogen within the proteins have a mass-absorption coefficient an order of magnitude greater. $X$ rays within this so-called water-window can be produced by focusing a laser onto a carbon target, producing a plasma that emits strongly at the $C^{5+}(33.7-\AA)$ and $C^{4+}(40.3-$ A) lines.

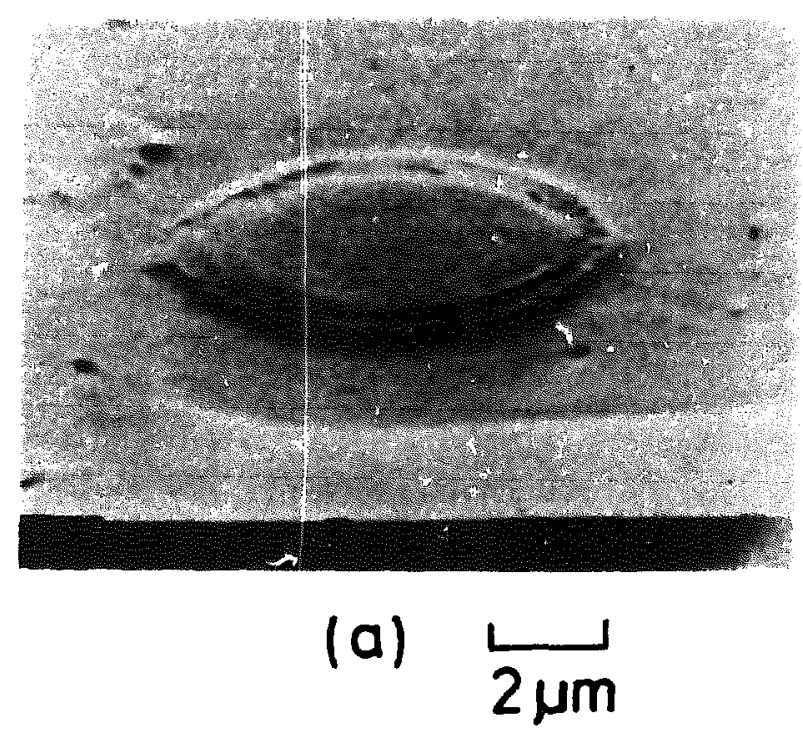

Some resilts of this work are shown in Fig. 12. The sample here is a red blood cell, recorded with a 1-ns $x$-ray exposure. The features observed in such images are well below the resolution obtainable with optical microscopes, and study of several botanical samples has revealed structure not observable using traditional transmission electron microscopy methods. Thus, LP $\mathrm{x}$ rays, which can probe living tissue before cell death occurs, show great potential in furthering our knowledge of the structure of living cells.

We have described above the use of LP $x$ rays for materials study using diffraction and for the study of biological systems using contact microscopy. These two areas of research have been brought closer together by the work of Frankel, Forsyth, and Yaakobi, who have studied x-ray diffraction from biological samples. Some of the most interesting work has involved the study of the purple membrane (PM) of the Halobacterium halobium. This membrane consists of a hexagonally packed, two-dimensional array of the protein bacterio-rhodopsin (BR), and is thus ideal for diffraction studies. Frankel and Forsyth (1979) used the resonance line of He-like chlorine (4.44 $\AA$ ) to create a quasi-monochromatic beam to diffract off the PM sample at low angles (the lattice constant of the membrane is at $\sim 63 \AA$ ). The resultant powder diffraction pattern showed

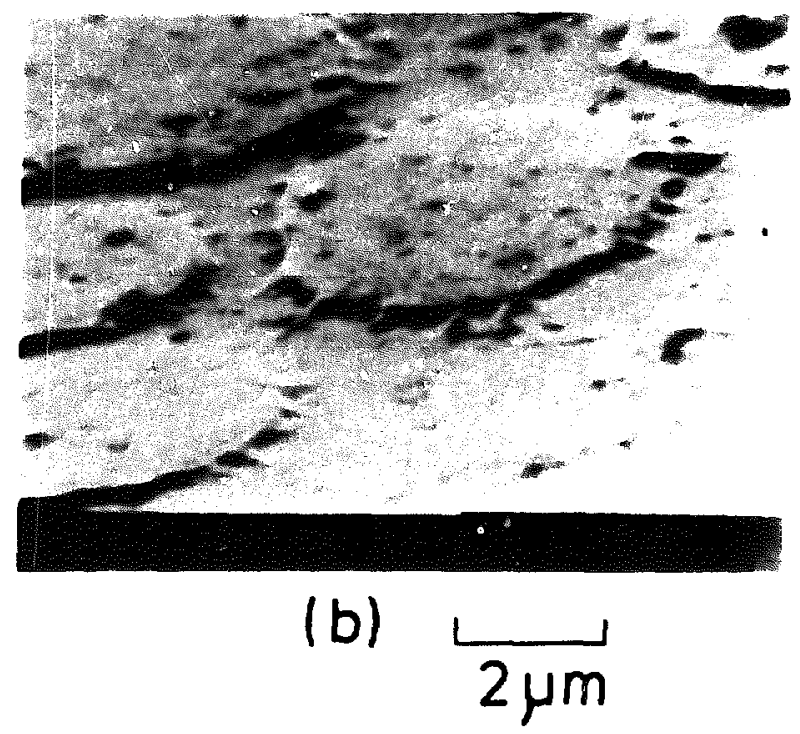

Fig. 12. Replicas of in vivo red blood cell (a), and cells attacked by melittir (b). (Results of work by M. Clague, University of Essex, U.K.) 
multiple-order reflections, with the highest order indicating a resolution of $\sim 7 \AA$ in the plane of the membrane.

The PM is a photobiological system: it has been shown that, upon illumination, the BR protein sets up a transmembrane hydrogen ion gradient within which there may be molecular changes. Frankel and Forsyth used the synchronization and single-shot ability of an LP $x$-ray source to probe a PM sample $1 \mathrm{~ms}$ after photoactivation. They saw considerable alterations in the resultant diffraction pattern, which they believe cannot simply be attributed to heating of the lattice but are indicative of photoactivity.

\subsubsection{X-Ray Lithography. The last few} years have seen an increased interest (mainly by industry) in the utilization of x-ray lithography for 1:1 shadowgraphy printing of a patterned mask in semiconductor circuit manufacturing. This interest results from the diffraction limitation on the resolution achievable with optical lithography, as well as problems associated with the small depth of field. X-ray lithography is the logical extention of the current optical lithography to submicron printed circuit manufacturing. For optical systems having very good aberration correction and employing a high degree of partial coherence, the minimum practical feature size can be as small as $0.6 \lambda / \mathrm{NA}$ (where NA is the numerical aperture of the optical system). The depth of focus of a diffraction-limited system is about $0.8 R^{2} / \lambda$ ( $R$ is the radius of the effective aperture). Clearly, for submicron feature sizes the depth of field is not larger than typical feature heights, necessitating the use of complex multilevel photoresist techniques. This expression also shows that shorter wavelengths produce a better combination of resolution and depth of focus, but the realization of a practical lithography system that utilizes short optical wavelengths is not easy. A typical high-performance reduction lens might utilize as many as six different glass types, each with a different index and dispersion to control the aberrations. Controlling the aberrations in such a lens requires that the position of each element in the assembly be carefully adjusted. Because the index of refraction of glass is also a function of temperature, the entire lens housing is generally temperature controlled to a fraction of a degree.
$\mathrm{X}$-ray lithography involves simple one-to-one shadow projection. (Spiller and Feder, 1977) of an $x$-ray-transparent membrane with absorber structures onto a resist-coated wafer. Between mask and wafer is a small gap (typically $20 \mu \mathrm{m}$ ) to protect the mask against mechanical damage. The resolution is then limited by the penumbral shadowing (except for a collimated beam) and also by diffraction. 'The wavelength region useful for the purpose of $x$-ray lithography is approximately 0.5 to $2.5 \mathrm{~nm}$. The longer-wavelength limit is determined mainly by the high absorption in the thin mask substrate; the shorter-wavelength limit is determined mainly by the decreasing absorption in the resist layer. $A$ variety of $x$-ray sources have been used in lithography development. $X$ ray tubes are beset by low fluence, requiring impracticably long exposure times. This is particularly so because the relatively large focal spot requires a large working distance. Synchrotron storage rings have the advantages of being powerful and collimated but are exceedingly expensive and complex for industrial application. Laserirradiated targets have the advantages of a very small source (hence, good resolution even at close distances) and the possibility of scaling to a highrepetition-rate industrial laser. Additional characteristics are the reproducibility of source positioning (to a few micrometers), almost complete freedom in choosing the target material (which affects tlie spectral characteristics), and mechanical stability in single-pulse operation. The typical shot-to-shot intensity variation of the total dose is about $\pm 10 \%$, but this can be greatly improved in mode-locked operation with feedback control.

$\mathrm{X}$-ray lithography using a LP source has been demonstrated and studied in the past years (Nagel et al., 1978; Mallozzi et al., 1979). More recently, it has been shown (Yaakobi et al., 1983) that a single 1-ns UV-laser pulse of 35-J energy can produce (shallow) exposure in PBS and COP resists. The corresponding $x$-ray dose on the resist was smaller, by more than a factor of 10 , than the nominal threshold exposure of these resists. The reason for this behavior is that the high peak irradiance of $x$ rays with these short $U V$-laser pulses is sufficent to induce resist ablation, so that the pattern is imprinted even without resist development. The shallowness of the ablation pattern can 
be improved by employing multilayer resist techniques. An example of relief structures obtained (Frenkel et al., 1987) in a single-layer resist by the Hannshire Instruments Company, using laserproduced x-ray lithography, is shown in Fig. 13. The vertical resist ridges ride over $0.5-\mu \mathrm{m}$-high silicon dioxide steps on the silicon substrate. The exposure was obtained with radation in the range of 8-20 $\AA$ using a boron nitride mask substrate with a 0.5- $\mu \mathrm{m}$ gold absorber. Submicron resolution and feature acuity are clearly evident.

\subsection{Particle Applications}

From the data presented in Table 3, one can see that a significant portion of the incident laser energy can be converted into protons and moderately heavy ions with energies greater than $1 \mathrm{MeV}$. This is enough energy to cause proton activation of nuclei and to result in the production of radioactive isotopes when a secondary target is bombarded.

With a moderate-size laser system producing around $1 \mathrm{~kJ}$ of energy in 1 ns (e.g., one module of the HELIOS laser system at the Los Alamos

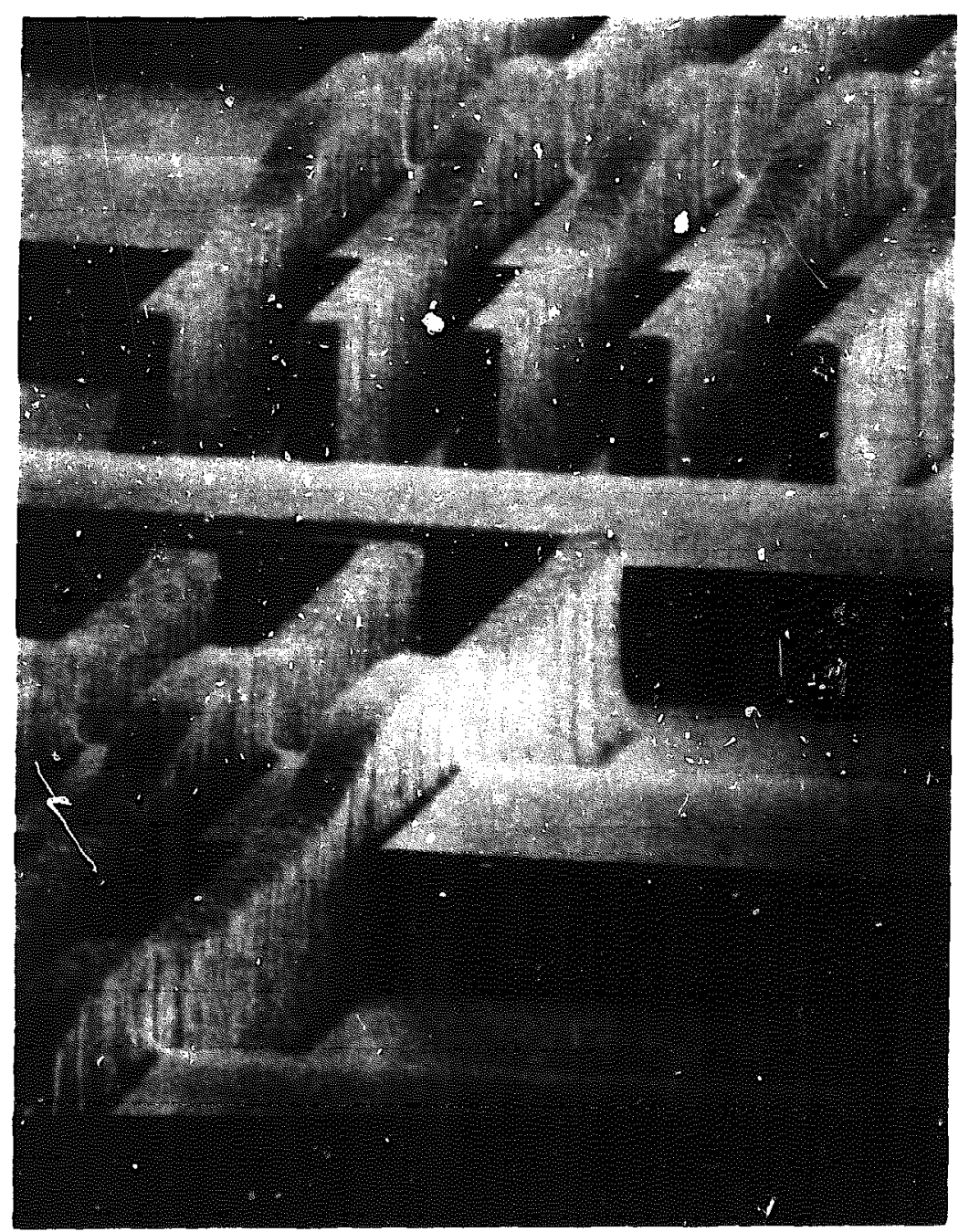

Fig. 13. Relief structure in AZ2400 resist obtained by laserproduced $x$-ray lithography (Frankel, et al., 1987). The resist ridges ride over 0.5 -micron-high silicon dioxide steps on a silicon substrate. (Courtesy of Hampshire Instruments, Inc.) 
National Laboratory), it should be possible to produce $150 \mathrm{~J}$ of $1-\mathrm{MeV}$ protons. An upgrade in laser technology might make possible a $1-\mathrm{Hz}$ firing rate; this in turn would result in a total average current of protons of about $100 \mathrm{mi}$ croamps. This amperage is comparable to the current available from cyclotrons. Many advances would be required to create a practical alternative to cyclotron-produced isotopes. These advances would include collimation of the ion blowoff to be compatible with the small sample size needed in isotope work (Obrien, 1987), protection of the target from low-energy ion debris, and thermal loading.

Another possible application for high-energy ion blowoff is plasma formation in magnetic mirror machines (Mayer, Berk, and Forslund, 1985). The stability in magnetic fusion devices is often discussed in relation to the parameter beta, which is the ratio of plasma particle nressure to magnetic field pressure. The plasma configuration in mirror machines is unstable at low beta but may be stable at high beta. It is essential in these systems to have rapid plasma formation and thus rapid arhievement of high-beta conditions. One method of producing the rapid plasma formation could be laser-produced fast-ion blowoff. Ion energies of the order of $100 \mathrm{keV}$, which can readily be produced by $\mathrm{CO}_{2}$ lasers (as shown in Table 3), are required in this application. Mayer, Berk, and Forslund (1985) have proposed an experiment that would help to determine the viability of this interesting new application of high-energy ions.

Finally, ion jets, as typified by Fig. 5, may be of value in simulating and studying astrophysical conditions (Forslund and Goldstone, 1985). The ion jets are reminiscent of galactic jets that have been identified in radio-wave photographs. In addition, it has been observed (Forslund and Goldstone, 1985) that there is a striking similarity between the normalized electromagnetic spectrum from a typical $\mathrm{CO}_{2}$ laser/target interaction and that emitted by the Crab Nebula. $\mathrm{CO}_{2}$ LPs have been observed to emit up to $0.1 \%$ of the incident laser energy as microwaves. Such surprising emission efficiency may be due to an enhancement in the expanding corona of those plasma waves that couple efficiently to microwave emission. A mechanism of this type is a strong candidate for explaining microwaves emitted from both galactic jets and from solar flares. A more detailed laboratory investigation of these processes could help us discriminate between the various theories of astrophysical microwave emission.

\section{LASER-PLASMA ACCELERATION OF PARTICLES}

\subsection{LASERS AND PLASMAS}

The maximum accelerating field that can be produced with current accelerator technology is of the order of $1 \mathrm{MeV} \mathrm{cm}^{-1}$. At field strengths greater than this, one encounters the problem of breakdown of the accelerator walls. To build the next generation of particle accelerators, one has two choices. The first is to use conventional technology and simply increase the size of the accelerator, with all the financial and logistical difficulties that this entails. The second is to develop new accelerator technologies. Ideally, these would have a larger accelerating field and would cost less per unit particie energy than today's accelerators.

In this section, one of the proposed new accelerators, namely the plasma beat-wave accelerator (Tajima and Dawson, 1979; Joshi et al., 1984), will be described. The basic idea is to use two lasers to generate a longitudinal space-charge wave in a plasma. The phase speed of this spacecharge wave must be close to the speed of light so that a relativistic particle will stay in phase with the electrostatic field of the wave long enough to be accelerated to high energy. Before describing this scheme in detail, however, it is instructive to consider why it might be desirable to incorporate lasers and plasmas in accelerator technology.

The main reason for using lasers and plasmas is the magnitude of the electric fields that can be produced. Specifically, the peak electric field in a laser is related to the laser irradiance by

$$
e E_{l}\left[\mathrm{eV} \mathrm{cm}^{-1}\right] \approx 27\left(I_{l}\left[\mathrm{~W} \mathrm{~cm}^{-2}\right]\right)^{1 / 2}
$$

so that an incident irradiance of $10^{16} \mathrm{~W} \mathrm{~cm}^{-2}$ corresponds to an electric field of approximately 
$2.7 \mathrm{GeV} \mathrm{cm} \mathrm{cm}^{-1}$. This is larger than the maximum accelerating field of conventional accelerators by more than three orders of magnitude! Unfortunately, however, the transverse polarization of these electric fields renders them unsuitable for direct particie acceleration. Any scheme to couple particles to the laser fields necessarily involves transverse particle acceleration and the resulting radiation losses. This suggests that waves with longitudinal electric fields should be used. One obvious candidate is the longitudinal space-charge wave in a plasma, in which the electrostatic field is generated by periodic density compressions and rarefactions of the plasma electrons. The natural angular frequency of this "plasma wave" is known as the plasma frequency and has a value of

$$
\omega_{p} \approx 5.6 \times 10^{4}\left(n_{e}\left[\mathrm{~cm}^{-3}\right]\right)^{1 / 2} .
$$

The maximum electrostatic field can be estiunated from Poisson's equation by setting the perturbed density equal to the backround density and taking the plasma wavevector to be consistent with the requirement that the phase speed of the plasma wave is close to the speed of light. The resulting electrostatic field is given by

$$
e E_{c}\left[\mathrm{eV} \mathrm{cm}^{-1}\right] \approx 0.97\left(n_{\mathrm{e}}\left[\mathrm{cm}^{-3}\right]\right)^{1 / 2}
$$

and is known as the cold wavebreaking field because it is the largest electrostatic field that can be produced before the field amplitude becomes double-valued (Dawson, 1959). In a plasma with a background density of $10^{18} \mathrm{~cm}^{-3}$, the cold wavebreaking field is approximately 0.97 $\mathrm{GeV} \mathrm{cm}^{-1}$, which is again larger than the maxium acclerating field of conventional accelerators by tiree orders of magnitude. This clearly illustrates one potential advantage of using plasmas. Another advantage is that a plasma is already ionized and so no further damage can be done to it by these large electric fields.

\subsection{PLASMA-WAVE GENERATION}

In the plasma beat-wave accelerator, the plasma wave is generated by the beating of two colinear laser beams (Rosenbluth and Liu, 1972). The radiation pressure of the lasers induces longitudinal plasma oscillations at the laser beat- frequency. If the incident frequencies are chosen so that the plasma wave is resonantly driven, the conservation of energy and momentum is manifested by the frequency and wavevector matching conditions

$$
\omega_{1}=\omega_{0}+\omega_{p}, \quad \overrightarrow{k_{1}}=\overrightarrow{k_{0}}+\overrightarrow{k_{p}}
$$

where the higher-frequency laser is denoted by the subscript 1 , the lower-frequency laser is denoted by the subscript 0 , and the plasma wave is denoted by the subscript $p$. It follows that the phase speed of the plasma wave can be expressed in terms of the incident frequencies and wavevectors as $\left(\omega_{1}-\omega_{0}\right) /\left(k_{1}-k_{0}\right)$. In an underdense plasma, in which the plasma-wave frequency and wavevector are much smaller than the incident frequencies and wavevectors, this is approximately equal to the group speed $d w_{l} / d k_{l}=c\left(1-\omega_{p}^{2} / \omega_{l}^{2}\right)^{1 / 2}$ of the light waves.

The plasma-wave amplitude evolves accordiıg to the equation

$$
\left(\frac{d}{d t}-i \frac{3}{16}\left|A_{p}\right|^{2}\right) A_{p}=\frac{1}{4} A_{1} A_{0},
$$

where time is measured in units of $\omega_{p}^{-1} \cdot A_{p}$ is the peak amplitude of the density fluctuation associated with the plasma wave, normalized to the background density. The corresponding electrostatic field is the product of this normalized density fluctuation and the cold wavebreaking field [Eq. (5)]. $A_{1}$ and $A_{0}$ are the peak "quiver" velocities of electrons (divided by the speed of light) in the laser fields and are related to the incident laser irradiances by

$$
A_{l}=8.5 \times 10^{-10} \lambda_{l}[\mu \mathrm{m}]\left(I_{l}\left[\mathrm{Wcm}^{-2}\right]\right)^{1 / 2} .
$$

In the linear regime, the plasma wave grows indefinitely. Eventually, however, the quiver velocity of electrons in the plasma-wave field becomes so large that the lowest-order relativistic corrections to the electron mass must be retained in the equations of motion. This results in a nonlinear reduction in the natural frequency of the plasma wave by an amount that is proportional to the square of the wave amplitude (Akhiezer and Polovin, 1956). As a result of this relativistic frequency shift, the plasma wave is driven out of pliase with the beating of the two light waves, and the plasma-wave 
anplitude saturates at the value

$$
A_{\text {max }}=\left(\frac{16}{3} A_{1} A_{0}\right)^{1 / 3} .
$$

If the interaction is allowed to continue, the plasma-wave energy is fed back into the light waves and the plasma-wave amplitude decreases accordingly.

In solving Eq. (6) for the saturated plasmawave amplitude [Eq. (7)], it was tacitly assumed that the incident laser amplitudes are constant. In reality, the incident laser pulses have finite pulse length $\tau_{I}$ and propagate through the plasma at their group speed. Consider the interaction of the light waves and the plasma wave at some arbitary point $\xi_{0}$ in the plasma. Initially, the plasma wave has only noise-level amplitude. At some time $\tau_{0}$, the leading edges of the laser pulses reach the position $\xi_{0}$. The plasma wave then starts to grow according to Eq. (6), with laser amplitudes that now depend on time. This growth continues until the plasma-wave amplitude reaches a value close to that predicted by Eq. (7) or until the trailing edges of the laser pulses pass by, whichever occurs first. For times later than $\tau_{0}+\tau_{l}$, the plasma wave oscillates freely with the amplitude it had at time $\tau_{0}+\tau_{l}$. To maximize the energy in the plasma wave, the laser pulse length must be tailored to coincide with the maximum plasma-wave amplitude, as shown in Fig. 14. This limits the energy in the incident laser pulses and, hence, the energy that can ultimately be delivered to the accelerated particles in one stage. Because the group speed of a plasma wave in a typical beat-wave plasma is essentially zero, the energy deposited in the plasma wave is left behind the laser pulses, which continually propagate into fresh plasma and continue the process anew.

The saturation time and saturated amplitude for a given pulse shape can be estimated by using the linear plasma-wave amplitude to determine the cumulative phase shift caused by the plasma nonlinearity. Imposing the condition that the cumulative phase shift equals $\pi / 2$ radians determines the saturation time and saturated amplitude as a function of the incident laser intensities and pulse shapes. The incident pulse lengihs can then be chosen to coincide with the maximum plasma-wave amplitude, as discussed above. For laser-beam amplitudes that grow linearly in time, the saturation time is given by

$$
\tau_{s}\left[\omega_{p}^{-1}\right] \approx 20\left(A_{1} A_{0}\right)^{-2 / 3}
$$

whereas the saturated amplitude is given by

$$
A_{\max } \approx 1.7\left(A_{1} A_{0}\right)^{1 / 3}
$$

In the above formulas, $A_{1}$ and $A_{0}$ denote the peak laser amplitudes.

This simple theory of plasma-wave generation is in good agreement with the results of computer simulations (Katsouleas et al., 1985; Forslund et al., 1985). Experimental verification was first. obtained by Joshi et al. (1985). The 9.6- $\mu \mathrm{m}$ $\left(A_{1} \approx 0.030\right)$ and $10.6-\mu \mathrm{m}\left(A_{0} \approx 0.015\right)$ lines of a

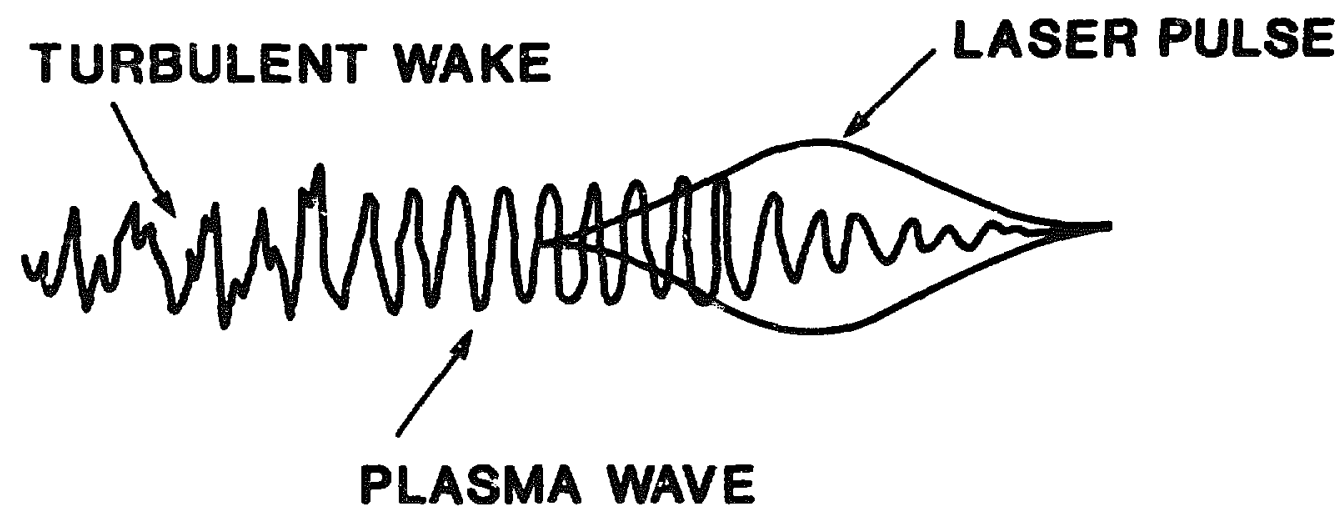

Fig. 14. Maximizing of the energy in the plasma wave through tailoring of laser pulsewidth. 
$\mathrm{CO}_{2}$ laser were used to resonanuly drive a plasina wave in a plasma of density $1.1 \times 10^{17} \mathrm{~cm}^{-3}$. The laser amplitudes were modeled as growing linearly in time for a duration of 1 ns. For these parameters, Eq. (6) predicts a maximum plasma-wave amplitude $A_{p}$ of approximately 0.08 at the middle of the laser pulse. By measuring the timeintegrated scattered light, an average amplitude $A_{p}$ of $0.01-0.03$ was inferred, in resonable agreement with the theoretical estimate. The corresponding electrostatic field was $3-10 \mathrm{MeV} \mathrm{cm}^{-1}$, which marked the first time a longitudinal field in excess of $1 \mathrm{MeV} \mathrm{cm}^{-1}$ had been produced in a controlled manner.

Behind the laser pulses, the wake of the plasma wave becomes turbulent, as shown in Fig. 14 . This can be due to the parametric decay instability, in which the nlasma wave decays into a secondary plasma wave and an ion-acoustic wave (Dubois and Goldman, 1965 and 1967; Silin, 1965) or the modulational instabiiity (Whitham, 1974; Pesme et al., 1987). These instabilities both involve ion motion and occur on a time scale longer than the electron time scale $\omega_{p}^{-1}$ by a factor of $\left(m_{i} / Z m_{e}\right)^{1 / 2}$, where $Z$ is the ionic charge. The modulational instability can also occur because of the relativistic nonlinearity in Eq. (6). Because the turbulent wake cannot be used for particle acceleration, it might be inferred that the energy in the wake of the plasma wave is wasted. This is not necessarily true. In practice, the presence of these plasma instabilites tends to limit the time for plasma-wave growth and beam loading (McKinstrie and Forslund, 1987). This is discussed in cetail in a later section.

In the preceding analysis, the self-consistent evolution of the light-wave amplitudes was not taken into account. Just as the beating of the incident light waves produces a resonant plasma wave at the difference frequency, the beating of the transverse electron quiver velocities with the plasma-wave density fluctuation produces oscillating currents at the sum and difference frequencies. In this way, a spectrum of colinear light waves is generated, with frequencies and wave vectors that differ from those of the incident waves by integral multiples of $\omega_{p}$ and $\vec{k}_{p}$, respectively. This nonlinear interaction can also be viewed as a series of three-wave processes in which a photon either decays into a lower-frequency photon and a plasmon or recombines with a plasmon to produce a higher-frequency photon. Notice that the total number of photons is conserved and that the number of plasmons is equal to the difference between the number of decay interactions and the number of recombination interactions. It follows that only a fraction, $\omega_{p} / \omega_{1}$, of the incident laser energy can be transferred to the plasma wave in the primary three-wave interaction (Manley and Powe, 1956). To increase the energy transfer to the plasma wave, and ultimately to the accelerated particles, the laser energy must be made to cascade "downwards" from the incident waves to their lower-frequency sidebands. Significant contributions to the current understanding of this process have been made by Cohen, Kaufman, and Watson, (1972), and by Karttunen and Salomaa. (1987). However, the extent to which the incident energy can be made to cascade to lower frequencies remains an active and important area of current research. The reader is referred to the review by McKinstrie and Batha (1987). If one assumes that most of the laser energy can be transferred to the plasma wave and that the rate of energy transfer is constant, then the pump-depletion length $L_{d}$ can be estimated from the requirement that the energy contained in the wake of the plasma wave is equal to the total energy that was originally contained in the laser pulses. Taking the plasmawave amplitude to be given by Eq. (9) and the laser pulse lengths to be given by $\mathrm{Eq}$. (8) yields

$$
L_{d}\left[c \omega_{p}^{-1}\right] \approx 4.6 \gamma_{\phi}^{2} A_{l}^{-2 / 3}
$$

for the case of equal-irradiance lasers. The Lorentz factor $\gamma_{\phi}$, associated with the phase speed of the plasina wave, is equal to $\omega_{l} / \omega_{p}$.

\subsection{PARTICLE INJECTION}

Just as a surfer must paddle to catch an ocean wave, an electron cannot be trapped and then accelerated by a plasma wave unless it has a certain minimum velocity parallel to the plasma wave. The corresponding minimum energy is determined by the requirement that in the wave frame the electron has insufficient linetic energy to ecape the plasma-wave potential well. The potential energy of an electron in the plasmawave field is shown in Fig. 15. Because of the 


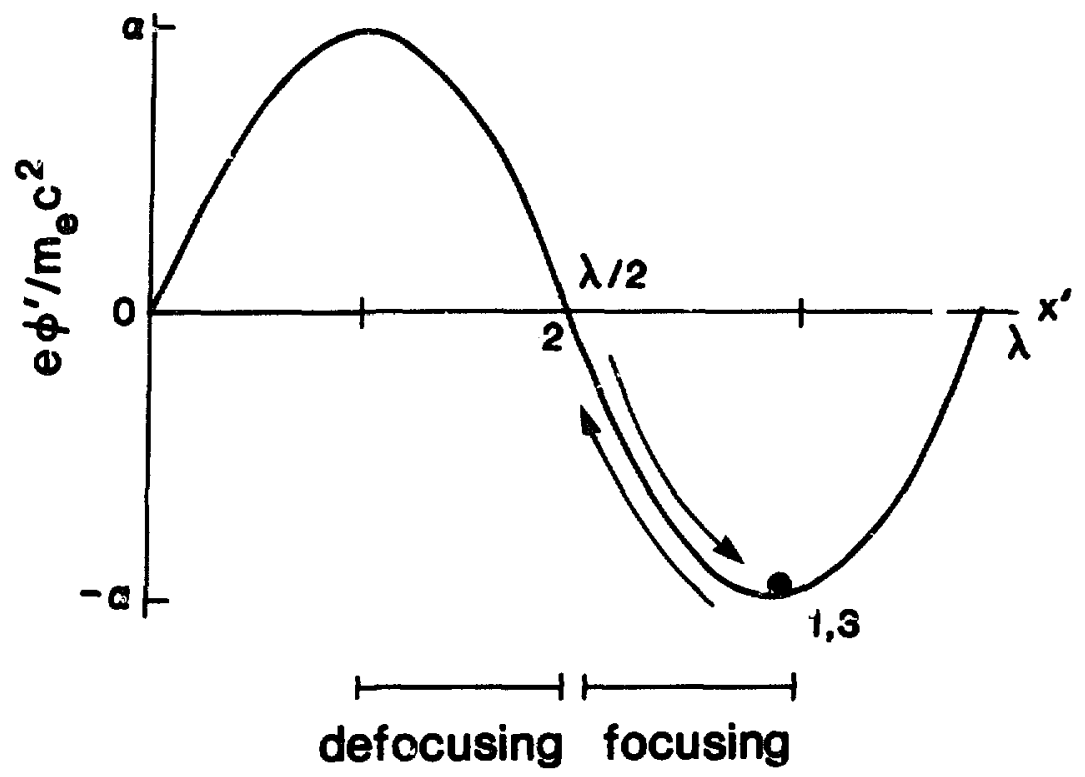

Fig. 15. Potential enargy of an electron in the plasma-wave field.

radial fields arising from the finite width of the plasma wave, only the quarter wavelength between point 1 and point 2 is both accelerating and focusing to a bunch of electrons (Katsouleas, 1986a). Consequently, an electron injected at point 1 must reach its turning point before it reaches point 2 if it is to be usefully accelerated. In terms of laboratory-frame quantities, the Lorentz factor corresponding to the minimum injection energy (Katsouleas et al., 1985) is given by

$$
\gamma_{i}=\gamma_{\phi}\left(1+\alpha-\beta_{\phi}[\alpha(2+\alpha)]^{1 / 2}\right),
$$

where

$$
\alpha=\gamma_{\phi} \beta_{\phi} A_{p}
$$

is the peak potential energy oi the electron in the wave frame, normalized to the electron rest-mass. This is also the average injection energy for electrons that are injected at random into the field of the plasma wave. The electron injection energy is plotted as a function of the plasma-wave amplitude in Fig. 16. For a typical plasma temperature of $200 \mathrm{eV}$, there is negligible trapping of electrons, which must therefore be externally accelerated before being injected into the field of the plasma wave. The injection energy for ions can also be obtained from Eq. (11) by multiplying $\alpha$ by $Z m_{e} / m_{i}$. Because the resulting value of $\alpha$ is much less than unity, $\gamma_{i}$ must then be approximately equal to $\gamma_{\phi}$. This effectively precludes ion acceleration.

On reaching point 2 , an electron reverses its wave-frame motion and is further accelerated until it reaches point 3. The final electron energy (Yan, 1986) is given by

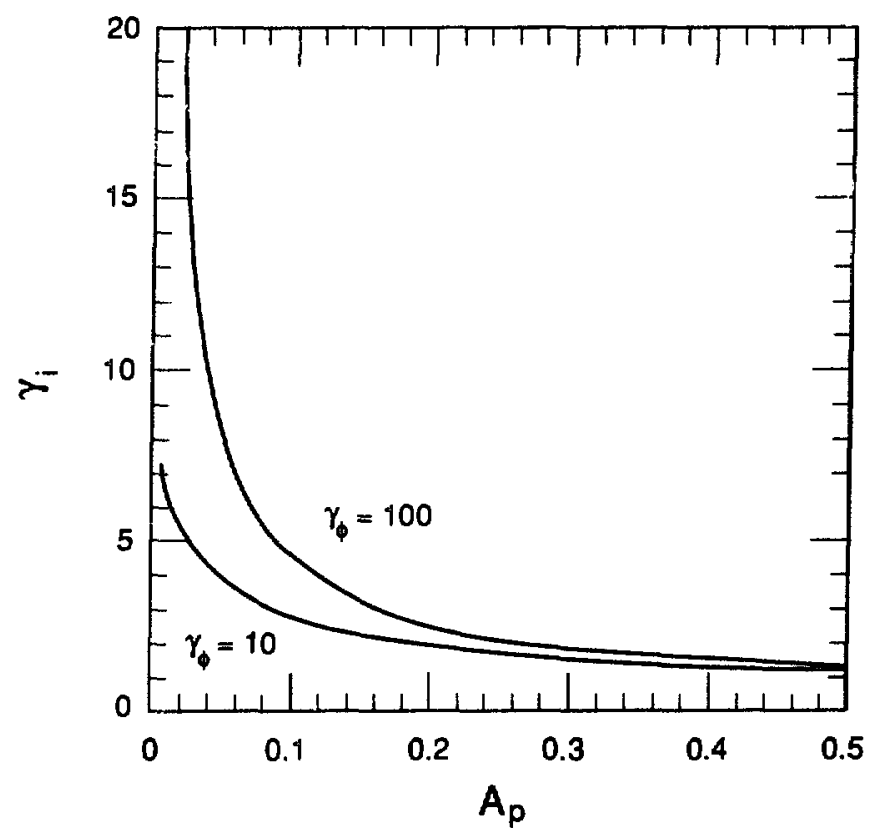

Fig. 16. Electron injection energy as a function of plasma-wave amplitude. 


$$
\gamma_{f}=\gamma_{\phi}\left(1+\alpha+\beta_{\phi}[\alpha(2+\alpha)]^{1 / 2}\right)
$$

From Eqs. (11) and (12), the energy gain is easily seen to be

$$
\Delta \gamma=2 \gamma_{\phi} \beta_{\phi}[\alpha(2+\alpha)]^{1 / 2}
$$

The gain in electron energy is plotted as a function of the plasma-wave amplitude in Fig. 17. For the high-gain limit in which $\alpha$ is much greater than unity, $\Delta \gamma$ is approximately equal to $2 \gamma_{\phi}^{2} A_{p}$. This high-gain limit can also be derived by a simple physical argument. As an electron moves from point 1 to point 2 , its laboratory-frame energy is increased from $\gamma_{i}$ to $\gamma_{\phi}$. This increase is much smaller than the total increase, which is of the order of $\gamma_{\phi}^{2}$, and can therefore be neglected. As the electron moves from point 2 to point 3 , the large c'ectric field necessary for high gain rapidly accelerates the electron to relativistic speeds in the wave frame. Consequently, the electron spends an equal amount of time at each point of the wave. The work done on the electron in the laboratory frame is therefore equal to $e\left\langle E_{p}\right\rangle L_{a}$, where $\left\langle E_{p}\right\rangle=2 E_{p} / \pi$ is the average of the electric field taken over one-quarter wavelength and the acceleration length $L_{a}$ is the distance travelled by the

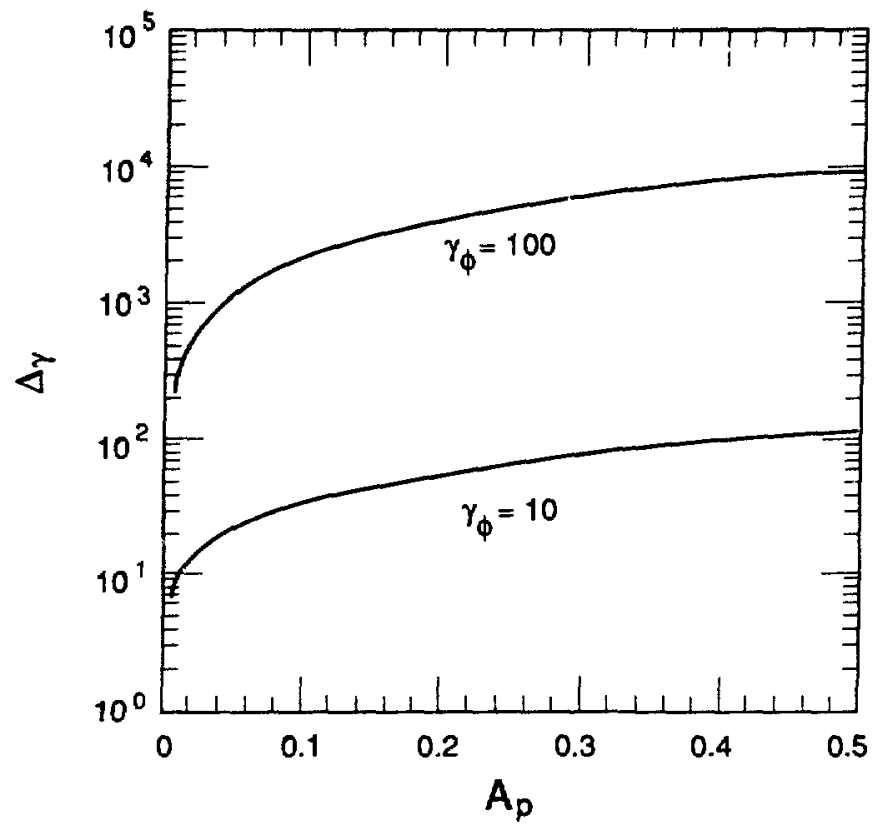

Fig. 17. Gain in electron energy as a function of plasma-wave amplitude. electron in the laboratory frame as it traverses one-quarter wavelength in the wave frame. Approximating the speed of the accelerated electron by the speed of light, the acceleration length is $\lambda_{p} /\left[4\left(1-\beta_{\phi}\right)\right] \approx \gamma_{\phi}^{2} \lambda_{p} / 2$. Rewriting the electric field in terms of $A_{p}$ gives $e\left\langle E_{p}\right\rangle L_{a} \approx 2 \gamma_{\phi}^{2} A_{p} m_{e} c^{2}$, in complete agreement with the high-gain limit of Eq. (13).

For easy comparison with the pump-depletion length [Eq. (10)], the acceleration length can also be written as

$$
L_{a}\left[c \omega_{p}^{-1}\right] \approx 3.1 \gamma_{\phi}^{2}
$$

It follows that the ratio $L_{a} / L_{d}$ is approximately equal to $0.67 A_{l}^{2 / 3}$. This ratio is an upper bound on the LP coupling efficiency $\eta_{c}$, defined as the fraction of incident laser energy that is transferred to the plasma wave during the acceleration phase.

Ebrahim et al. (1986) were tije first to observe the acceleration of injected electrons. In their experiment, the 9.6- $\mu \mathrm{m}\left(A_{1} \approx 0.054\right)$ and 10.6$\mu \mathrm{m}\left(A_{0} \approx 0.060\right)$ lines of a $\mathrm{CO}_{2}$ laser were used to resonantly drive a plasma wave in : plasma of density $1.1 \times 10^{17} \mathrm{~cm}^{-i}$. For these parameters, theory predicts a maximum plasma-wave amplitude $A_{p}$ of approximately 0.16 and a corresponding maximum electrostatic field of approximately $50 \mathrm{MeV} \mathrm{cm}^{-1}$. The electrons were produced by irradiating an aluminum slab with an auxiliary high-intensity $\mathrm{CO}_{2}$ laser. The energy of the electrons obtained in this manner was $0.5-1.0 \mathrm{MeV}$, which is greater than the minimum injection energy [Eq. (11)] of $0.46 \mathrm{MeV}$. Measurements indicated that electrons injected at $0.6 \mathrm{MeV}$ were accelerated to $2.0 \mathrm{MeV}$. Because the resonant plasma region was only $0.15 \mathrm{~cm}$ long, this implied that the average electrostatic field was $10 \mathrm{MeV} \mathrm{cm}^{-1}$, in resonable agreement with the theoretical estimate.

\subsection{BEAM LOADING}

In calculating the energy gain for a single electron, the self-generated electrostatic field can safely be neglected. However, in determining the energy gain of a bunch of electrons, the selfgenerated field $E_{b}$ is crucially important. The 
standard approach to this problem is to determine the electrostatic field produced by a single electron as it propagates through the plasma. The electrostatic field produced by a bunch of electrons can then be obtained by linear superposition.

Because the group speed of a plasma wave in a typical beat-wave plasma is essentialy zero, there can be no electrostatic field ahead of the electron, regardless of whether or not it is propagating at a relativistic speed. Behind the electron, a sinusoidal electrostatic field is produced, with a phase speed that is equal to the speed of the electron.

Providing the amplitude of the plasma wave is not too close to the cold wavebreaking limit, the amplitudes of the nonlinear harmonics are much less than the amplitude of the fundamental and the plasma wave is essentially sinusoidal. If an infinitesimally short bunch of electrons is injected into the plasma-wave field before the plasma wave becomes turbulent, the electrostatic field of the plasma wave can be completely cancelled. The laser energy that would have gone into the wake of the plasma wave is now absorbed by the electron bunch. By equating the relevant electrostatic fields, the number of accelerated electrons per unit cross-sectional area of the plasma wave, denoted by $\sigma_{0}$, is easily found to be

$$
\sigma_{0}\left[\mathrm{~cm}^{-2}\right] \approx 5.4 \times 10^{5} A_{p}\left(n_{e}\left[\mathrm{~cm}^{-3}\right]\right)^{1 / 2}
$$

For a plasma of background density $10^{18} \mathrm{~cm}^{-3}$ and a plasma-wave amplitude $A_{p}$ of, for example, 0.2 , this is approximately $10^{14}$ electrons per $\mathrm{cm}^{2}$.

The beam-loading efficiency $\eta_{b}$ is defined as the plasma-wave energy absorbed by the electron bunch divided by the plasma-wave energy in the absence of the electron bunch. The case of an infinitesimally short bunch described above corresponds to a beam-loading efficiency of $100 \%$. Unfortunately, however, this is only achieved at the expense of a $100 \%$ spread in the energy of the accelerated electrons. This is because the first electron in the bunch feels the full accelerating field $E_{p}$, whereas the last electron in the bunch feels the superposed field $E_{p}+E_{b}$, which is equal to zero. Because the reduction in accelerating field is linear in the areal number density $\sigma$ of the bunch the fractional energy spread is simply equal to $\sigma / \sigma_{0}$.
The fraction of plasma-wave energy absorbed by the bunch is quadratic in the areal number density of the bunch, and is readily shown to be

$$
\eta_{b}=\left(\sigma / \sigma_{0}\right)\left[2-\left(\sigma / \sigma_{0}\right)\right] .
$$

Equation (16) illustrates the tradeoff between the total number of accelerated particles and the fractional energy spread for infinitesimally short bunches.

In principle, it is possible to reduce the energy spread without significantly lowering the beamloading efficiency by appropriately shaping the electron bunch (Katsouleas et al., 1987). This possibility is an active area of current research. However, it is not clear whether such precise shaping of the electron bunch, over distances that are much smaller than the plasma wavelength, is technologically feasible. A realistic electron bunch has a density distribution that is approximately Gaussian. Calculations show that, for particles within one standard deviation of the peak density, the accelerating field is approximately equal to onethird of the plasma-wave field and varies by approximately $10 \%$. The corresponding energy gain is therefore about one-third of the ideal value predicted by Eq. (13). It can also be shown that the number of accelerated electrons is about threequarters of the number suggested by Eq. (15).

\subsection{LASER REQUIREMENTS}

The previous sections have shown how lasers can be used to generate a large-amplitude plasma wave with a phase speed that is close to the speed of light. The electrostatic field of this plasma wave can be of the order of $1 \mathrm{GeV} \mathrm{cm} \mathrm{cm}^{-1}$. Equation (13) shows that, in the high-gain limit, the energy gain of a suitably injected electron is proportional to $\left(\omega_{l} / \omega_{p}\right)^{2}$. A high energy gain requires this ratio to be large. For a fixed energy gain, the acceleration length [Eq. (14)] is proportional to $c \omega_{p}^{-1}$. This length can be mimimized by the use of high-density plasmas, which, in turn, requires the use of short-wavelength lasers. A particular example is a KrF laser (Jensen, 1987), which oscillates at $0.25 \mu \mathrm{m}$ with an inherent bandwidth of about $1 \%$. The design specifications for a plasma 
beat-wave accelerator, based on a $\mathrm{KrF}$ amplifier, are given in Table 4. The electron energy gain is approximately $1.0 \mathrm{GeV}$ per stage in a distance of approximately $12 \mathrm{~cm}$. Thus, by use of multiple staging, it is theoretically possible to accelerate electrons to an energy of $10 \mathrm{TeV}$ in a distance of the order of $1 \mathrm{~km}$. This distance is two orders of magnitude less than for the proposed superconducting super collider (SSC).

There are still many questions to be answered before the plasma beat-wave accelerator can be considered a practical device. First and foremost are the still-open questions of laser efficiency and LP coupling efficiency. From Table 4 and the preceding analysis, any reasonable estimate of laser efficiency leads to a wall-plug efficiency of only a few percent. However, even if lasers prove to be too inefficient for the next generation of accelerators, they are an important intermediate step because of their demonstrated ability to produce large-amplitude, monochromatic plasma waves.

In this brief review, only the basic, onedimensional theory of the plasma beat-wave accelerator has been described. For a more detailed account of this and other plasma-based acceleration schemes, the interested reader is referred to the articles by Katsouleas (1986b), Joshi et al. (1987), Mori (1987), and Bingham and Evans (1988).

\begin{tabular}{ll}
\hline Table 4. Case Study for a KrF Laser \\
\hline$\lambda_{L}[\mu \mathrm{m}]$ & $2.5 \times 10^{-1}$ \\
$\Delta \lambda_{L}[\mu \mathrm{m}]$ & $2.5 \times 10^{-3}$ \\
$I_{L}\left[\mathrm{Wcm}{ }^{-2}\right]$ & $1.0 \times 10^{17}$ \\
$\tau_{L}[\mathrm{ps}]$ & 9.8 \\
spot size $[\mu \mathrm{m}]$ & 25 \\
laser energy $[J]$ & 11.6 \\
$n_{e}\left[\mathrm{~cm}^{-3}\right]$ & $1.8 \times 10^{18}$ \\
$e E_{p}\left[\mathrm{MeV} \mathrm{cm}^{-1}\right]$ & 360 \\
$\Delta T_{e}[\mathrm{MeV}]$ & $1.0 \times 10^{3}$ \\
$L_{a}[\mathrm{~cm}]$ & 12 \\
\hline \hline
\end{tabular}

\section{LASER PULSED-POWER SWITCHING}

Recent progress in our understanding of the operation and preformance of laser-triggered spark gaps has been made possible, by the availability of reliable pulsed lasers with wavelengths covering the range from the ultraviolet (UV) to the infrared (IR) (Guenther and Bettis, 1985). At the same time, fundamental studies of the interaction of laser radiation with dielectric gases have been improved through the use of high-bandwidth recorders, such as streak cameras (Dougal and Williams, 1984), coupled with new optical and electrical sensors (Chang et al., 1985). The new impetus for developing laser-triggered spark gaps is based on the need to synchronize numerous high-voltage events to within several nanoseconds. This need is exemplified by the PBFA-II lightjon-beam fusion accelerator located at Sandia National Laboratories in Albuquerque, New Mexico (Turman et al., 1985) (Fig. 18). The PBFA-II has 36 5-MV gas dielectric switches that are routinely triggered with a total timing spread of 10 ns.

Because of its importance in low-jitter highvoltage triggering, we will restrict our attention to UV-laser-triggered $\mathrm{SF}_{6}$-insulated spark gaps. Where appropiate, comparisons will be made to the preformance of similar IR-triggered switches. The reader is referred to review articles by Guenther and Bettis $(1978 ; 1985)$ for in-depth information on other types of laser-triggered spark gaps.

Laser-triggered switch systems should have the following desirable characteristics:

(1) low jitter;

(2) mininum sensitivity of switch closure time, i.e. delay, to switch voltage and laser energy;

(3) low switch prefire rate;

(4) low required laser energy;

(5) low maintenance;

(6) high reliability; and

(7) efficient laser, beam-transport, and opticalalignment systems.

Low-jitter triggering with minimum change in switch delay with switch voltage has been demonstrated with UV lasers in $\mathrm{SF}_{6}$-insulated switches 


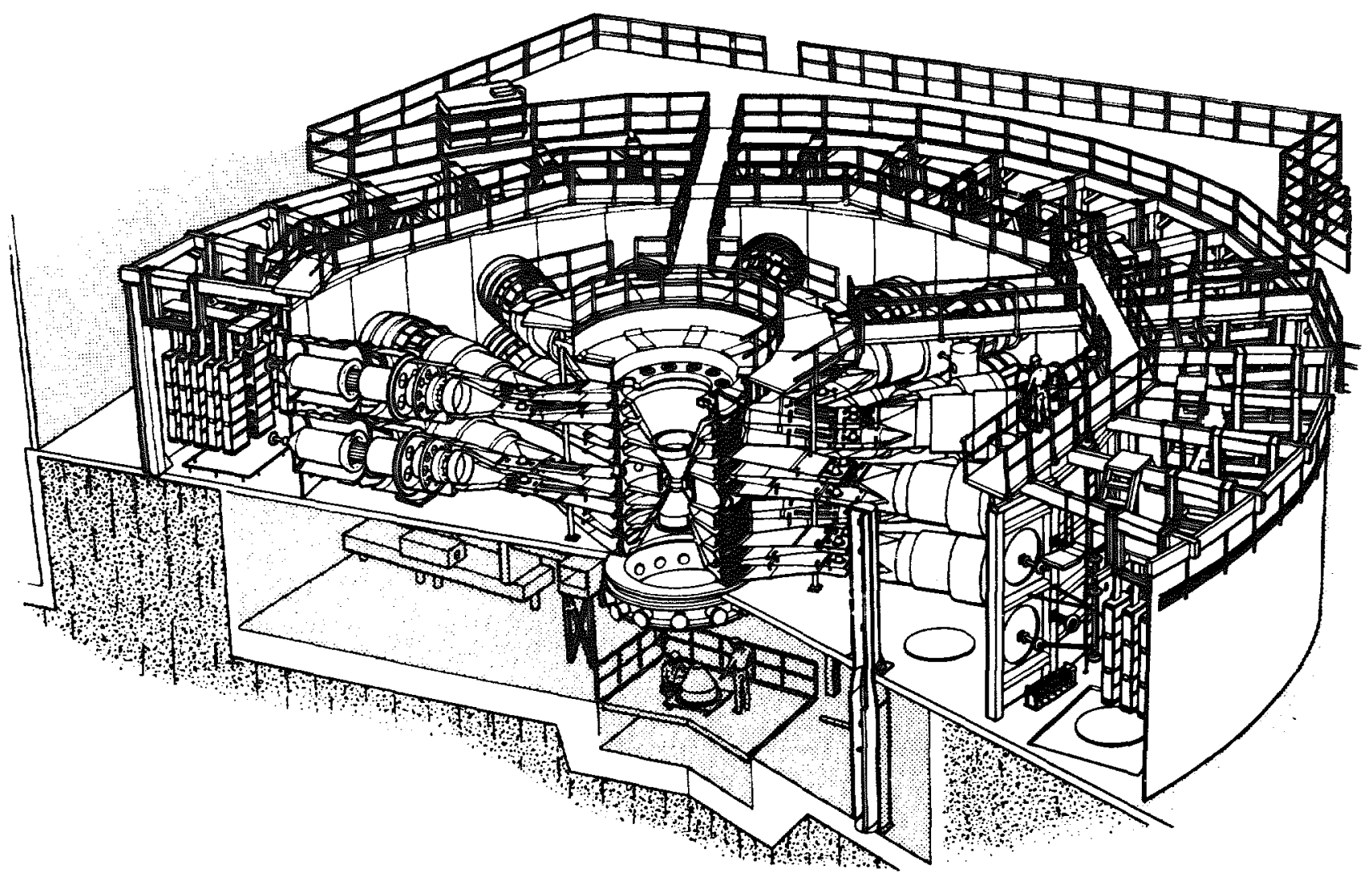

Fig. 18. PBFA-II light-ion beam fusion accelerator at Sandia National Laboratories.

on numerous occasions (Rapoport et al., 1980; Woodworth et al., 1982; Hamil and Smith, 1983; Woodworth et al., 1984; Humphreys et al., 1985). The need for low jitter is obvious when numerous high-voltage switching operations must be synchronized or the switch needs to be synchronized with another fast event. The change in the gap closure time with switch voltage gives the necessary uniformity of switch-to-switch charge voltage for multiple gas switches to achieve low jitter. The prefire rate is reduced when the switch is triggered well below its self-breakdown voltage. Switch maintenance is also reduced because there is no trigger electrode-typically the first componet to fail in an electrically triggered switch. However, the optics in a laser-triggered system rnust be kept free of the debris and caustic gases produced within the switch. Experience with numerous high-voltage switches has shown that this requires a proper purge-gas system and an optical design with the final optical element placed at least $50 \mathrm{~cm}$ from the laser breakdown spark (Hamil et al., 1985a; 1985b). The laser, delivery optics, and alignment system must be economical, simple, reliable, and easy to use.

Rapoport et al. (1980) first described the use of laser radiation from a krypton flouride $(\mathrm{KrF})$ excimer laser to trigger an $\mathrm{SF}_{6}$-insulated switch with subnanosecond jitter. In this experiment, $\mathbf{S F}_{6}$ gas was used because of its low UV-breakdown threshold and its high dc-breakdown strength. A 7-mJ, 20-ns-long pulse at a wavelength of $248 \mathrm{~nm}$ from the $\mathrm{KrF}$ laser was focused into the center of a $0.5-\mathrm{cm}, 80-\mathrm{kV}$, dc-charged, high-voltage gap. The laser was focused through a 0.25 -cm-diameter hoie in the high-voltage electrode. Subnanusecond jitter at $80 \%$ of the self-breakdown voltage was measured. Empirical studies showed that switch jitter was reduced when the incoming laser beam was very close to grazing the entrance hole in the high-voltage electrode. This effect may be associated with the particular electrode geometry and 
the diameter of the laser entrance hole relative to the gap spacing. In the geometry of Rapoport et al. (1980), the entrance hole diameter was equal to half the gap spacing. The unilluminated distance from the laser beam to the edge of the hole could therefore have a significant effect on the measured switch jitter. Unpublished experiments (Hamil and Ramirez, 1987) performed at Sandia National Laboratories with two identical 2.5MV switches of the Rimfire design (Humphreys et al., 1985) demonstrated that entrance hole effects can be minimized. In the Sandia experiments, the nanosecond rms-trigger-jitter between the two switches was not affected when one of the lasertrigger beams was moved from the center to the edge of the entrance hole. In these Rimfire experiments, the ratio of the electrode spacing to the entrance hole diameter was 6 , as compared with 2 in the experiments of Rapoport et al. (1980). The Sandia results illustrate the importance of the gap spacing to entrance hole diameter ratio in designing laser-triggered switch systems.

Prior to the work of Rapoport et al. (1980), laser triggering experiments using IR or visiblewavelength lasers had focused the laser near the far electrode to generate an electrode-based plasma (Guenther and Bettis, 1978). It has been suggested (Horii et al., 1970; Guenther and Bettis, 1978) that UV radiation from the electrode plasma serves as the source of the channel that eventually closes the gap. Dougal and Williams $(1984 ; 1986)$ showed that the channel that eventually closes the gap preferentially follows the path of the laser beam. However, subnanosecond jitter is only achieved with IR and visible-wavelength lasers at pulse energies at least an order of magnitude greater than those required for UV-laser triggering.

The UV-laser work at Sandia National Laboratories has heen aimed at developing pulsecharged megavolt-class switches that can be triggered with nanosecond jitter using minimum laser energy. In that effort, a 5-MV multistage switch was triggered reliably with $10 \mathrm{~mJ}$ of laser energy to achieve 1.5-ns jitter (Humphreys et al., 1985), a 2.5-MV multistage switch was triggered with as little as $5 \mathrm{~mJ}$ of laser energy and 1-ns jitter (Hamil, 1987), and a 3-MV single-gap switch was triggered with $20 \mathrm{~mJ}$ of laser energy to achieve 2-ns jitter (Hamil and Smith, 1983).
Several authors (Guenther and Bettis, 1978; Woodworth et al., 1982; Woodworth et al., 1984; Guenther and Bettis, 1985; Humphreys et al., 1985) have observed the effect of laser pulse length on switch delay and jitter. With an IR-laser trigger source, the pulse-length dependency is quite distinct. The switch delay and jitter become quite large as soon as the switch delay exceeds the laser pulse length. The UV-laser-trigger data do not always show this very abrupt increase in jitter and delay when the laser pulse terminates before switch closure (Woodworth et al., 1984). Recent UV-laser trigger experinents (Hamil and Smith, 1983) performed at $3 \mathrm{MV}$ across an 11-cm gap showed that laser pulse-length effects can be minimized. These unpublished experiments achieved lower jitter and delay with a short 2-ns-long pulse than with a 20-ns-long pulse of equal energy. Additional factors such as laser beam quality, laser pulse shape, spark formation time, switch geometry, and f-number of the optical system appear to be important in determining the performance of UV-laser-triggered spark gaps.

Dielectric breakdown of the $\mathrm{SF}_{6}$ gas by the incident laser pulse is believed to influence the performance of IR- and UV-laser-triggered high-voltage switches. Evidence for this comes from measurements of the temporal development of the laser breakdown spark in a flowing gas cell containing $\mathrm{SF}_{6}$ (Hargis, 1983). The incident laser pulse, the transmitted laser pulse, and the optical sidelight emitted by the breakdown spark were measured with a temporal resolution of about 0.5 ns using fast photodiodes coupled to transient digitizers. A representative single-pulse result for the dielectric breakdown of 1000-Torr $\mathrm{SF}_{6}$ by a $30-\mathrm{mJ}$ laser pulse at $266 \mathrm{~nm}$ is shown in Fig. 19. Depletion of the incident laser pulse (determined by subtracting the transmitted and incident laser pulse shapes) and optical emission from the breakdown spark occur at the same time. The time delay between the leading edge of the incident laser pulse and the leading edge of the breakdown sidelight pulse increases as the incident laser pulse energy decreases. The total laser energy required to initiate dielectric breakdown of the $\mathrm{SF}_{6}$ was determined by integrating the incident laser pulse shape up to the time corresponding to the onset of the breakdown sidelight pulse. Figure 20 shows that the laser energy required to initiate dielectric 


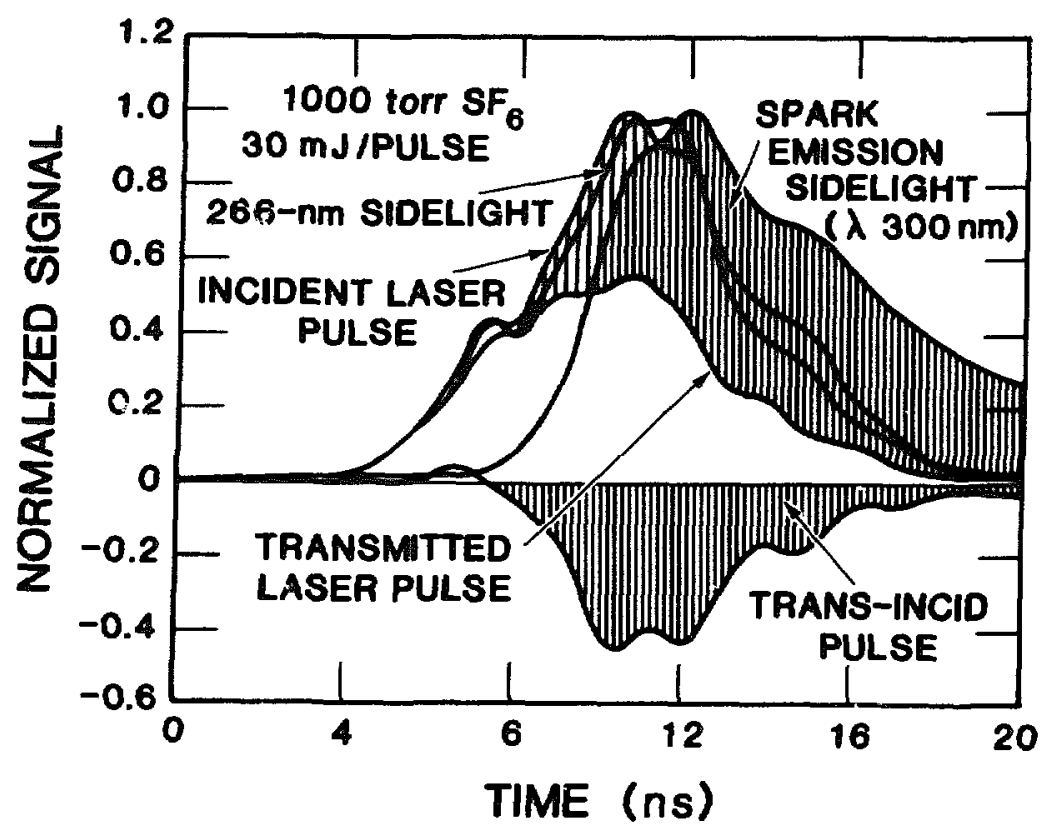

Fig. 19. Temporal development of 266-nm laser spark emission.

breakdown is independent of the time delay. This result suggests the importance of laser fluence in determining the onset of dielectric breakdown in $\mathrm{SF}_{6}$.

Similar data for the onset of dielectric breakdown in $\mathrm{SF}_{6}$ by an IR laser at $1064 \mathrm{~nm}$ show that the spark initiated by the IR radiation develops on a much slower time scale and persists for a longer time than the spark initiated by the UV laser at $266 \mathrm{~nm}$ (Hargis, 1983). A fixed amount of laser energy is also required to initiate dielectric breakdown with the IR-laser pulse. Because the break-

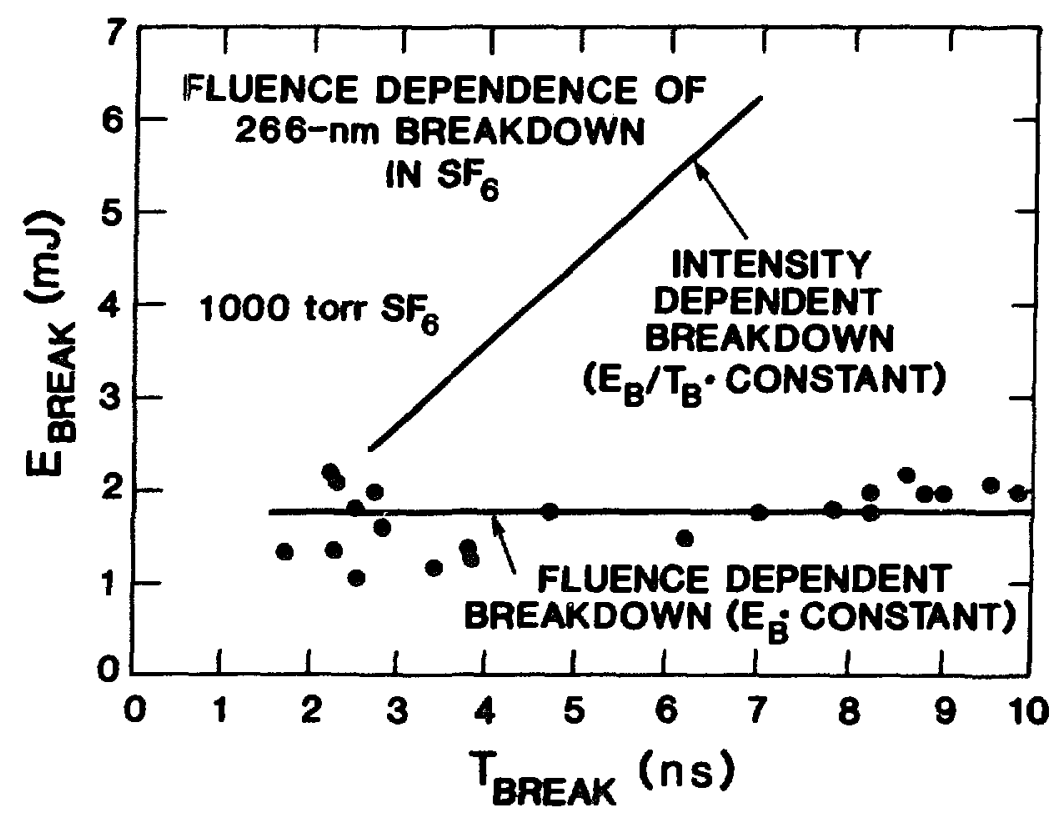

Fig. 20. Fluence dependence of $266-\mathrm{nm}$ breakdown in $\mathrm{SF}_{8}$. 
down spark initiated by the 1064-nm laser pulse develops on a much slower time scale, the time at which dielectric breakdown occurs is more susceptible to fluctuations in the laser pulse shape-resulting in increased jitter in the formation of the laser spark.

Optical emission from the laser-initiated breakdown spark in $\mathrm{SF}_{6}$ provides information on species playing a role in the laser-induced breakdown process. This is illustrated in Fig. 21 by the time-resolved emission spectra shown of the breakdown spark in 1000-Torr $\mathrm{SF}_{6}$ initiated by a $30-\mathrm{mJ}$ laser pulse at $266 \mathrm{~nm}$. The $\mathrm{SF}_{6}$ was flowing in a gas cell at a rate sufficient to change the gas in the breakdown volume between each laser pulse. The spectra in Fig. 21 were recorded on a scanning monochromator and with a tine resolution of 20 ns. Zero delay corresponds to the incidence of the 266-nm laser pulse in the breakdowil spark volume. The most striking features of these data are the line-broadened atomic fluorine emission peaks at wavelengths between 650 and $750 \mathrm{~nm}$. Decreased broadening of the atomic fluorine lines at late times after the initiation of the breakdown spark is readily apparent. The spectral resolution in Fig. 21 is not sufficent to resolve the individual contributions from.Stark, pressure, and Doppler broadening effects. Higherresolution data should make it possible to measure the temporal cependence of the Stark width of the broadened lines. Recently measured Stark broadening parameters (Mar, Czernichowski, and Chapelle, 1986) can then be used to estimate the average electron density in the breakdown spark volume.

It is instructive at this point to discuss the dielectric breakdown of $\mathrm{SF}_{6}$ and the related optical sidelight when the breakdown spark is initiated by two laser pulses at different wavelengths. The time scale for the formation of the breakdown spark in $\mathrm{SF}_{6}$ is faster in the UV at $266 \mathrm{~nm}$ than in the IR at $1064 \mathrm{~nm}$ (Hargis, 1983). If both the UV and IR pulses of light are focused together in a gas cell containing $S_{6}$, the seed electrons produced by the UV pulse can be heated by the IR pulse through an inverse bremsstrahlung mechanism (Zel'dovich and Razier, 1965; Morgan, 1975). Figures 22(a) and 22(b) clearly show an enhanced emission at wavelengths between $300 \mathrm{~nm}$ and $700 \mathrm{~nm}$ from the breakdown spark volume when the UV and IR pulses of light overlap each other temporally and spatially. The enhanced emission is attributed to IR heating of the seed electrons created by the UV pulse and/or an increase in the average electron density by the addition of the IR radation. Unlike the early results of Evseenko et al. (1979), which showed a reduced threshold for gas breakdown in a two-frequency laser field at wavelengths of $1064 \mathrm{~nm}$ and $532 \mathrm{~nm}$, the breakdown threshold at $266 \mathrm{~nm}$ is not affected by the presence of the $1064-\mathrm{nm}$ radation. The very bright spark created by the addition of the IR radiation was expected to be far superior to the faint spark produced $b_{j}$ the relatively lowenergy UV pulse when used to trigger a highvoltage gap. Figure 23 shows the characteristic

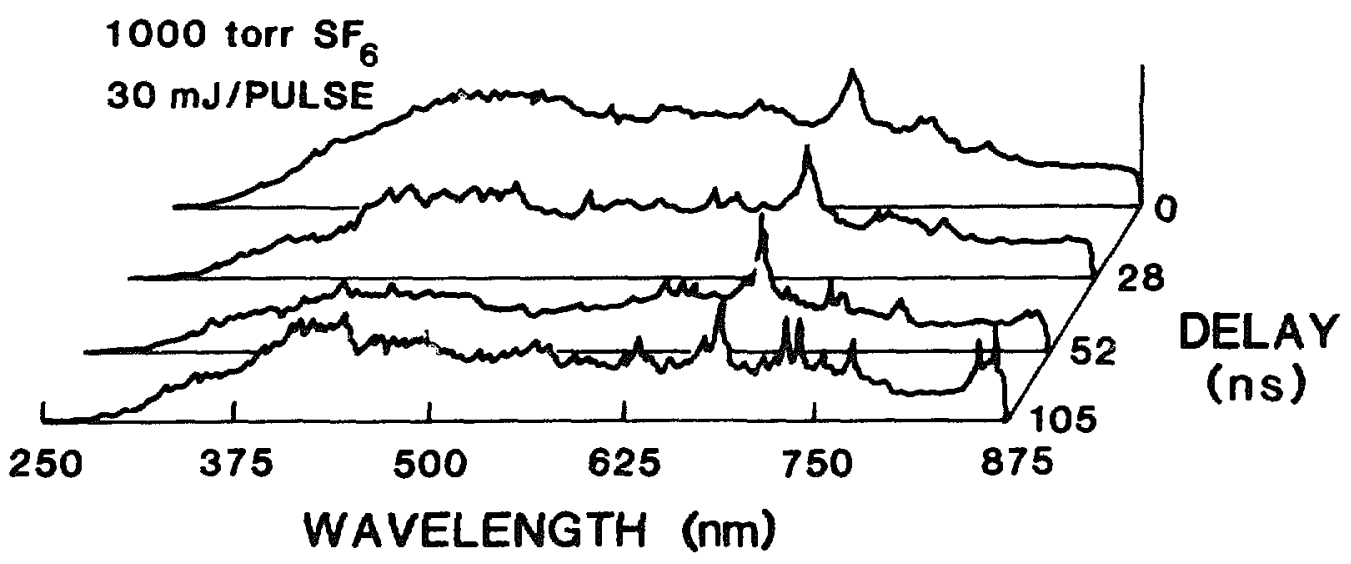

Fig. 21. Laser breakdown spectra in $\mathrm{SF}_{6}$. 

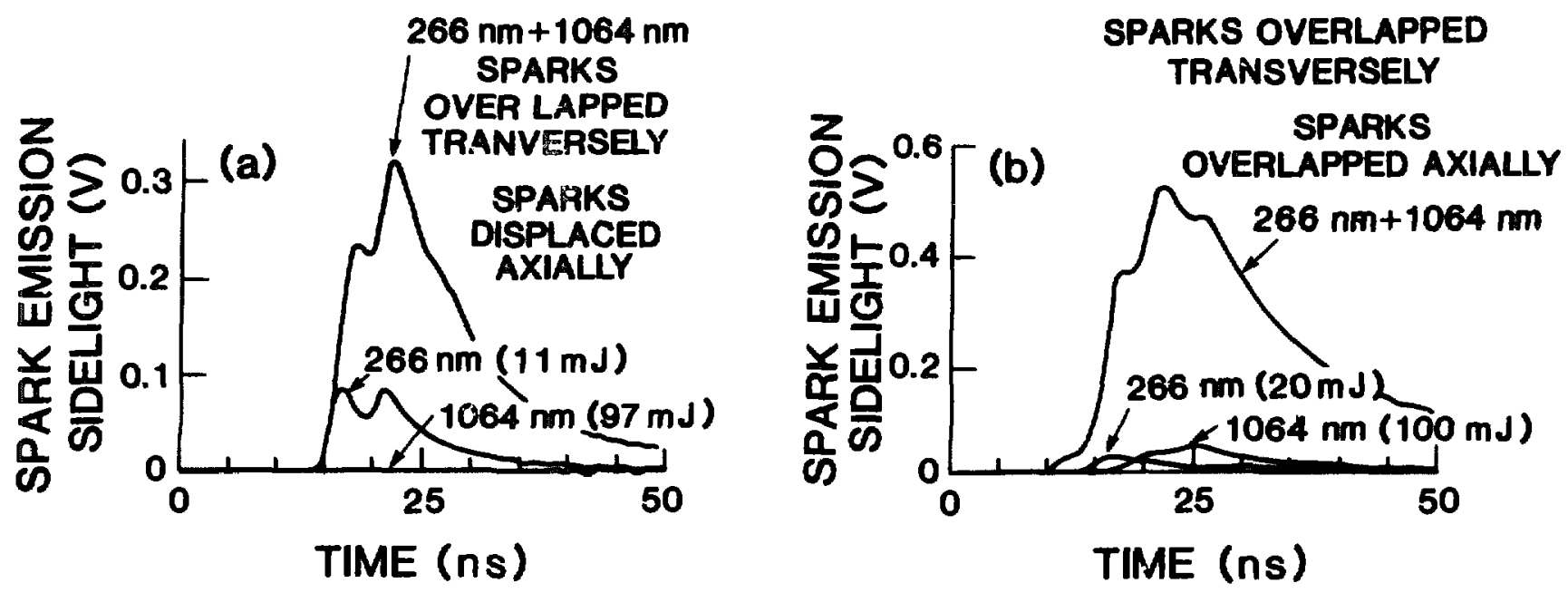

Fig. 22. Multifrequency laser breakdown in $\mathrm{SF}_{\mathrm{a}}$.

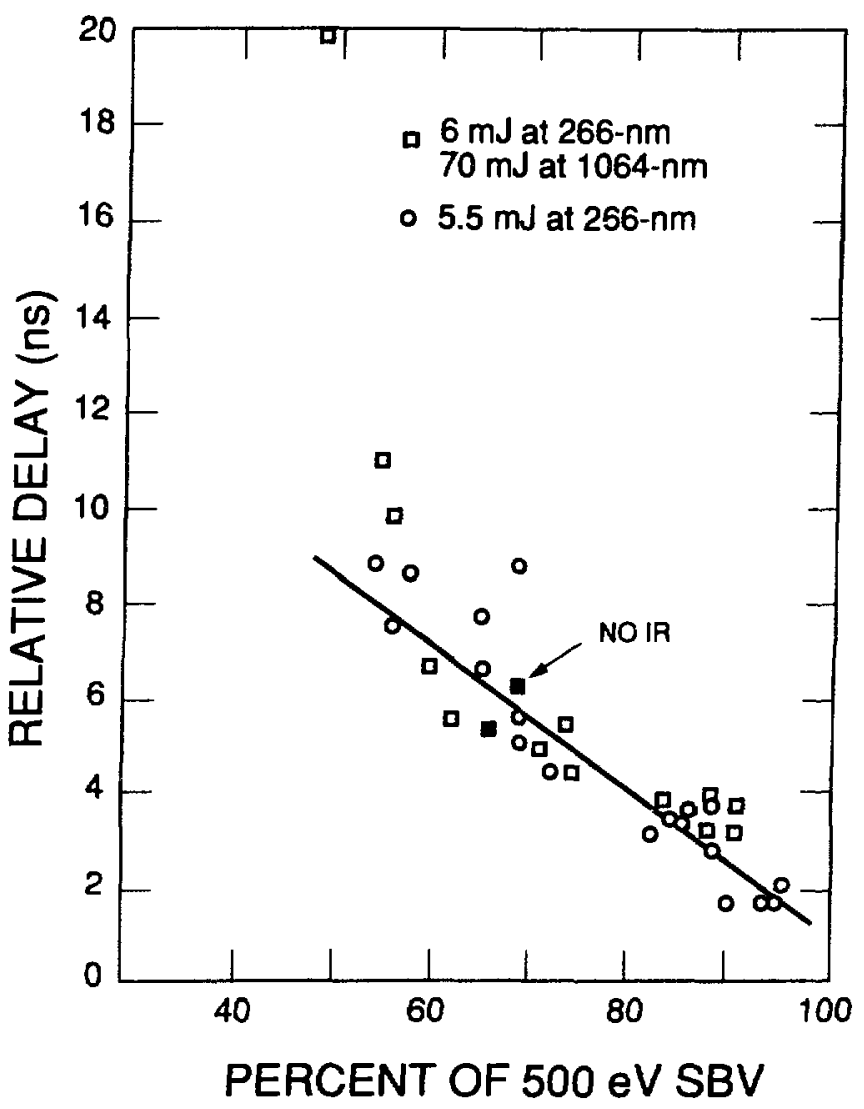

Fig. 23. Multifrequency versus UV triggering of a $50-\mathrm{kV}$ switch. 
breakdown curve for multifrequency triggering vs pure UV-laser triggering of a 0.5-MV gap. Note that there is no difference in jitter or delay even though the IR-laser pulse energy is more than ten times the UV-laser pulse energy. At this writing, we still do not fully understand the underlying physics involved in this phenomenon. The UV pulse alone seems to determine the performance of the switch.

The results presented in this brief section illustrate the progress made in understanding the operation and preformance of IR- and UV-lasertriggered spark gaps. New insights provided by fundamental studies of dielectric breakdown should lead to improved switch designs optimized for laser triggering. This goal, however, requires the development of comprehensive models incor- porating multiphoton effects in order to more fully understand the complex phenomena involved in the dielectric breakdown of $\mathrm{SF}_{6}$.

\section{ACKNOWLEDGMENTS}

The authors would like to thank Barukh Yaakobi for supplying material for the section on x-ray lithography and P. D. Goldstone, T. Katsouleas, W. B. Mori, and Y. T. Yan for useful discussions related to laser-particle acceleration. We would also like to acknowledge the careful editing and proofreading of Brian Thompson, Peggy Atencio, Kim Serna, and Jerry Weber and the excellent artwork of Susan Carlson and Cindy Boone. 


\section{REFERENCES}

Akhiezer, A. I., and Polovin, R. V. (1956). Theory of wave motion of an electron plasma, Sov. Phys. JETP, 30: 696.

Anderholm, N. C. (1970). Laser-generated stress waves, Appl. Phys. Lett., 16: 113.

Bingham, R., and Evans, R. G. (1988). Physics of beat-wave accelerators, to be published in Plasma Phys. and Controlled Fusion.

Birkhoff, G. D. (1958). The passage of fast electrons through matter, Handbuch der Physik $X X X I V$ (S. Flugge, Ed.), p. 53.

Bradley, D. K. (1985). Ph.D. Thesis, Imperial College of Science and Technology, University of London (Unpublished).

Burgeat, J. and Taupin (1968) Acta Crystallogr. A, 24: 99.

Burnett, N. H., Enright, G. D., Avery, A., Loen, A., and Kieffer, J. C. (1984). Time resolved $\mathrm{K}$ spectra in high intensity laser target interaction, Phys. Rev. A, 29: 2294.

Chang, J., Allen, G., Filter, W. F., Lockwood, G. J., and Neyer, B. T. (1985). Photonic diagnostics and pulsed power research, in Digest of Technical Papers, 5th IEEE Pulsed Power Confrence, Arlington, Virgina, June 10-12, 1985 (M. F. Rose and P. J. Turchi, Eds.), p. 1.

Cohen, B. I., Kaufman, A. N., and Watson, K. M. (1972). Beat heating of a plasma, Phys. Rev. Lett., 29: 581.

Dawson, J. M. (1959), Nonlinear electron oscillations in a cold plasma, Phys. Rev., 113: 383.
Dougal, R. A., and Williarns, P. F. (1984). Fundamental processes in laser-triggered electrical breakdown of gases, J. Phys. D 17: 903.

Dougal, R. A., and Williams, P. F. (1986). Fundamental processes in laser-triggered electrical breakdown of gases: Unconventional geometries, J. Appl. Phys., 60: 4240.

DuBois, D. F., and Goldman, M. V. (1965). Radiation-induced instability of electron plasma oscillations, Phys. Rev. Lett., 14: 544.

DuBois, D. F., and Goldman, M. V. (1967). Parametrically-excited plasma fluctuations, Phys. Rev., 164: 207.

Eason, R. W., Bradley, D. K., Kilkenny, J. D., and Geaves, G. N. (1984). Improved laserEXAFS studies of aluminin foil, $J$. Phys. $C$, 17: 5067 .

Eason, R. W., Cheng, P. C., Feder, R., Michette, A. G., Rosser, R. J., O'Neill, F., Owadano, Y., Rumsby, P. T., Shaw, M. J., and Turcu, I. C. E. (1986). Laser X-ray microscopy, Opt. Acta, 33: 501.

Ebrahim, N. A., Martin, F., Bordeur, P., Heighway, E. A., Matte, J. P., Pepin, HI., and Lavigne, P. (1986). First observations of acceleration of injected electrons in a laserplasma beat-wave experiment, in Proceedings of the 1986 Linear Accelerator Conference, Stanford Linear Accelerator Center, Stanford, California, June 2-6.

Evseenko, V. P., Mitsuk, V. E., and Soldatova, I. V. (1979). Reduced threshold for optical gas breakdown in a two-frequency field, Sov. Tech. Phys. Letí., 5: 329. 
Fairand, B. P., Wilcox, B. A., Gallagher, W. J., and Williams D. N. (1972). Laser shock-induced microstructural and mechanical property changes in 7075 aluminum, J. Appl. Phys., 43: 3893.

Feldman, U., Doschek, G. A., Nagel, D. J., Cowan, R. D., and Whitlock, R. R. (1974). Satellite line spectra from laserproduced plasmas, Astrophys. J., 192: 213.

Forslund, D. W., and Brackbill, J. (1982). Magnetic-field-induced surface transport on laser-irradiated foils, Phys. Rev. Lett., 48: 1614.

Forslund, D. W. and Goldstone, P. D. (1985). Photon impact, high energy plasma physics with $\mathrm{CO}_{2}$ lasers, Los Alamos Science, 12: 2.

Forslund, D. W., Kindel, J. M., Mori, W. B., Joshi, C., and Dawson, J. M. (1985). Two-dimensional simulations of single-frequency and beat-wave laser-plasma heating, Phys. Rev. Lett., 54: 558.

Forster, E., Goetz, K., Schafer, K., and Zimmer, W. D. (1984). Laser generated plasma as a source for real time studies in x-ray crystal research, Lasers and Particle Beams, 2: 167 and 187.

Frankel, R. D., Drumheller, J. P., Kaplan, A. S., and Lubin, M. J. (1987). Proceedings of Microelectronice Seminar INTERFACE-86 (Eastman Kodak), p. 82.

Frankel, R. D., and Forsyth, J. M. (1979). Nanosecond x-ray diffraction from biological samples with a laser-produced plasma source, Science, 204: 622 .

Graham, R. A., Nelson, F. W., and Benedick, W. B. (1965). Piezoelectric current from shock-Ioaded quartz-A submicrosecond stress gaugn, J. Appl. Phys., 36: 1775.

Guenther, A. H., and Bettis, J. R. (1978). Laser triggering of high voltage switches, $J$. Phys. D., 11: 1577.
Guenther, A. H., and Bettis, J. R. (1985). Recent advances in optically controlled discharges, in Digest of Technical Papers, Proceedings of the 5th IEEE Pulsed Power Conference, Arlington, Virginia, June 10-12, 1985 (M.F. Rose and P.J. Turchi, Eds.), p. 45.

Hamil, R. A. (1987). Private communication.

Hamil, R. A., and Smith, D. L. (1983). Laser triggered switch results from a frequency quadrupled Nd: YAG, in Laser, Proceedings of the 4th IEEE Pulsed Power Conference (M.F. Rose and T.H. Martin, Eds.), p. 447.

Hamil, R. A., and Ramirez, J. J. (1987). Private communication.

Hamil, R. A., Seamons, L. O., Schanwald, L. P., and Gerber, R. A. (1985a), Optical system for triggering PBFA II, in Digest of Technical Papers, 5th IEEE Pulsed Power Conference, Arlington, Virginia, June 10-12, 1985 (M. F. Rose and P. J. Turchi, Eds.), p. 803.

Hamil, R. A., Seamons, L. O., Gerber, R. A., Adams, R. C., Woodworth, J. R., Blackburn, R. S., and Sweatt, W. C. (1985b). Lasertrigger system design for PBFA II, in Digest of Technical Papers, 5th IEEE Pulsed Power Confrence, Arlington, Virgina, June 10-12, 1985 (M. F. Rose and P. J. Turchi, Eds.), p. 807 .

Hargis, p. J. (1983). Spectral and temporal evolution of laser breakdown in $\mathrm{SF}_{6}, \mathrm{Bull}$. Am. Phys. Soc., 28: 186.

Hauer, A. (1976). Interaction of x-rays and acoustic waves in solids, $P$ h.D. Thesis, University of Rochester, published by University Microfilms.

Hauer, A., Goldman, R., Kristal, R., Yates, M. A., Mueller, M., Begay, F., VanHulsteyn, D., Mitchell, K., Kephart, J., Oona, H., Stover, E., Brackbill, J., and Forslund, D. (1984). Suprathermal electron generation 
transport and deposition in $\mathrm{CO}_{2}$ laser irradiated targets, in Laser Interaction and Related Plasma Phenomena Vol. 6 (H. Hora and G. H. Miley, Eds.), Plenum Pub. Co., New York, p. 479.

Horii, K., Noguchi, T., and Yano, M. (1970). Spark discharge triggered by a pulse laser beam, in IEEE International Conference on Gas Discharges: IEEE Conference Publication No. 90 , p. 6.

Humphreys, D. R., Penn, K. J., Cap, J. S., Adams, R. G., Seamen, J. F., and Turman, B. N. (1985). Rimfire: A six megavolt lasertriggered gas switch for PBFA II, in Digest of Technical Papers, 5th IEEE Pulsed Power Conference, Arlington, Virginia, June 10-12, 1985, (M. F. Rose and p. J. Turchi, Eds.). p. 262

Jackson, J. D. (1975). Classical ElectroDynamics (2nd ed.), Wiley, New York, p. 643.

Jensen, R. (1987) . KrF for fusion: An overview of laser issues, Fusion Technology, 11: 481.

Joshi, C., Mori, W. B., Katsouleas, T., Dawson, J. M., Kindel, J. M. and Forslund, D. W. (1984). Ultrahigh-gradient particle acceleration by intense laser-driven plasma density waves, Nature, 311: 525.

Joshi, C., Clayton, C. E., Darrow, C., and Umstadter, D. (1985). Experimental study of beat-wave excitation of high phase-velocity space-charge waves in a plasma for particle acceleration, in Laser Acceleration of Particles (C. Joshi and T. Katsouleas, Eds.), AIP Conference Proceedings No. 130, American Institute of Physics, New York, p. 99.

Joshi, C., Katsouleas, T., Clayton, C. E., Mori, W. B., Kindel, J. M, and Forslund D. W. (1987). Experimental, theoretical and computational studies of the plasma beat-wave accelerator at UCLA, in Advanced Accelerator Concepts (F. E. Mills, Ed.), AIP Conference Proceedings No. 156, American Institute of Physics, New York, p. 71.
Karttunen, S. J., and Salomaa, R. R. E. (1987). Beat-wave generation of plasma waves for particle acceleration, IEEE Trans. Plasma Sci., PS-15: 134.

Katsouleas, T. (1986a), Physical mechanisms in the plasma wake-field accerator, Phys. Rev. A, 33: 2056.

Katsouleas, T. (1986b), Laser acceleration of particles, in High-Intensity Laser Processes, SPIE, 664: 2 .

Katsouleas, T., Joshi, C., Dawson, J. M., Chen, F. F., Clayton, C. E., Mori, W. B., Darrow, C., and Umstadter, D. (1985). Plasma accelerators, in Laser Acceleration of Particles (C. Joshi and T. Katsouleas, Eds.), AIP Conference Proceedings No. 130, American Institute of Physics, New York, p. 63.

Katsouleas, T., Wilks, S., Chen, P., Dawson, J. M., and Su, J. J. (1987). Beam loading in plasma accelerators, Particle Accelerators, 22: 81.

Kincaid, B. M., and Eisenberger, (1975). Synchrotron radiation studies of K-edge photo absorption spectra, Phys. Rev. Letî., 34: 1361.

Klar, B., and Rusticelli, F. (1973). Dynamical neutron diffraction by curved crystals in the laue geometry, Nuovo Cimento, 13B: 249.

Lunney, J. G., Dobson, p. J., Hares, J. D., Tabatabaei, S. D., and Eason, R. W. (1986). Time resolved x-ray diffracticn from silicon during pulsed laser annealing, Opt. Comm., 58: 269.

Mallozzi, p. J., Epstein, H. M., and Schwerzel, R. E. (1979), Advances in X-Ray Analysis, Plenum, New York, p. 267.

Mallozzi, p. J., Schwerzel, R. E., Epstein, H. M., and Campbell, B. E. (1979). Laser EXAFS: Laboratory EXAFS with a nanosecond pulse of laser-produced $\mathrm{x}$-rays, Science, 206: 353 . 
Manley, J. M., and Rowe, H. E. (1956). Scme general properties of nonlinear elements--Part I. General energy relations, Proc. IRE, 44: 904.

Mar, S., Czernichowski, A., and Chapelle, J. (1986). Experimental stark parameters for some spectral lines in $\mathrm{SF}_{6}$ plasma, $J$. Phys. $D$, 19: 621 .

Matthews, D. L., Campbell, E. M., Ceglio, N. M., Hermes, G., Kauffman, R., Koppel, L., Lee, R., Manes, K., Rupert, V., Slivinsky, R., Turner, R., and Ze., F. (1983). Characterization of laser produced plasma $x$-ray sources, J. Appl. Phys., 54: 4260.

Mayer, F. J., Berk, H. L., and Forslund, D. W. (1985). A laser-init:ated, energetic ion, high beta mirror plasma, Comm. on Plasma Phys. and Cont. Fusion, 9: 139.

McKinstrie, C. J., and Batha, S. H. (1987). Energy cascading in the beat-wave accelerator, in New Developments in Particle Acceleration Techniques (S. Turner, Ed.), Centre Européen de la Recherche Nucléaire, Geneva, p. 443.

McKinstrie, C. J., and Forslund, D. W. (1987). The detuning of relativistic Langmuir waves in the beat-wave accelerator, Phys. Fluids, 30: 904.

Michette, A. C., Chen, p. C., Eason, R. W., Feder, R., O'Neill, F., Owandano, Y., Rosser, R. J., Rumsby, P., and Shaw, M. J. (1986). Soft $\mathrm{x}$-ray contact microscopy using laser plasma sources, J. Phys. D, 19: 363.

Morgan, C.G. (1975). Laser induced breakdown of gases, Rep. Prog. Phys., 38: 621.

Mori, W. B. (1987). Theory and simulations on beat-wave excitation of relativistic plasma waves, $\mathrm{Ph} . \mathrm{D}$. Thesis, University of California at Los Angeles, published by University Microfilms, Ann Arbor, Michigan.

Nagel, D. J., Peckerar, M. C., Whitlock, R. R., Grieg, J. R., and Pecheack, R. E.
(1978). Submicrosecond x-ray lithography, Electron. Lett., 14: 781.

Obrien, H A. (1987). Private communication.

Pesme, D., Karttunen, S. J., Salomaa, R.R.E, Laval, G., and Silvestre, N. (1987). Modulational instablilty in the beat-wave generation, in New Developments in Particle Acceleration Techniques (S. Turner, Ed.), Centre Européen de la Recherche Nucléaire, Geneva, p. 418.

Phillion, D. W., and Hailey, C. J. (1986). Brightness and duration of $x$-ray line sources irradiated with 0.53 micron laser light at 60 and 120 picosecond pulse duration, Phys. Rev. $A, 34: 4886$.

Pirri, A. N. (1977). Theory for laser simulation of hypervelocity impart, Phys. Fluids, 20: 221.

Priedhorsky, W., Lier, D., Day, R., and Gerke, D. (1981). Hard $x$-ray measurements of 10.6micron laser-irradiated targets, Phys. Rev. Lett., 47: 1661.

Rapoport, W. R., Goldhar, J., and Murry, J. R. (1980). KrF laser-triggered $\mathrm{SF}_{\mathrm{s}}$ spark gap for low jitter timing, IEEE Transitions on Plasma Science, PS-8 (3): 167.

Rosenbluth, M. N., and Liu, C. S. (1972). Excitation of plasma waves by two laser beams, Phys. Rev. Lett., 29: 701.

Sayers, D. E., Stern, E. A., and Lytle, F. W. (1971). New technique for investigating noncrystalline structures: Fourier analysis of the extended $x$-ray absorption fine structure, Phys. Rev. Lett., 27: 1204.

Sheffield, S. A., and Fisk, G. A. (1983). in High Speed Photography, Videography, and Photonics (D. L. Paisley, Ed.), Proc. SPIE, 427: 193.

Sheffield, S. A., and Fisk, G. A. (1984). in Shock Waves in Condensed Matter (J. R. Asay, 
R. A. Graham, and G. K. Straub, Eds.), NorthHolland, New York, p. 243.

Silin, V. P. (1965). Parametric resonance in a plasma, Sov. Phys. JETP, 21: 1127.

Spiller, E., and Feder, R. (1977). Topics in Applied Physics: X-Ray Optics (H. J. Queisser, Ed.) (Springer, Berlin).

Tajima, T., and Dawson, J. M. (1979). Laser electron acceleration, Phys. Rev. Lett., 43: 267.

Turman, B. N., Martin, T. H., Neau, E. L., Humphreys, D. R., Bloomquist, D. D., Cook, D. L., Goldstein, S. A., Schneider, L. X., McDaniel, D. H., Wilison, J. M., Hamil, R. A., Barr, G. W., and VanDevender, J. P. (1985). PBFA II, A $100 \mathrm{TW}$ pulsed power driver for the inertial confinement fusion program, in Digest of Technical Papers, 5th IEEE Pulsed Power Conference, Arlington, Virginia, June 10-12, 1985 (M. F. Rose and P. J. Turchi, Eds.), p. 155.

Wark, J. S., Hauer, A., and Kilkenny, J. D. (1986). Studies of $x$-ray switching and shuttering techniques, Rev. Sci. Instrum., 57: 2168.

Wark, J. S., Whitlock, R. R., Hauer, A., Swain, J. E., and Salone, P. J. (1987). The study of shock launching in silicon using pulsed $x$-ray diffraction, Phys. Rev. B, 35: 9391.

Whitham, G. B. (1974). Linear and Nonlinear Waves, Wiley, New York, p. 485.

Woodworth, J. R., Frost, C. A., and Green, T. A. (1982). UV laser triggering of high-voltage gas switches, J. Appl. Phys., bi3: 4734.
Woodworth, J. R., Hargis, P. J., Jr., Pitchford, L. C., and Hamil, R.A. (1984). Laser triggering of a $500-\mathrm{KV}$ gas-filled switch: A parametric study, Appl. Phys., 56: 1382.

Yaakobi, B., Bourke, P., Conturie, Y., Delettrez, J., Forsyth, J. M., Frankel, R. D., Goldman, L. M., McCrory, R. L., Seka. W., Soures, J. M., Burek, A. J., and Deslattes, R. E. (1981). High $\mathrm{x}$-ray conversion efficiency with target irradiation by a frequency tripled Nd:glass laser, Opt. Comm., 38: 196.

Yaakobi, B., Kim, H., Soures, J. M., Deckman, H. W., and Dunsmuir, J. (1983). Submicron x-ray lithography using laser-produced plasma as a source, Appl. Phys. Lett., 43: 686.

Yaakobi, B., Frankel, R. D., Forsyth, J. M., and Soures, J. (1986). Laser generated x-rav source for time-resolved biological and materials structure stucies, in Structural Biological Applications of $X$-Ray $A b$ sorption Scattering and Diffraction, Academic Press, New York, p. 331.

Yan, Y. T. (1986). Interaction of relativistic electrons and radiation, Ph.D. Thesis, University of California at Los Angles, published by University Microfilms, Ann Arbor, Michigan, p. 92.

Yang, L. C. (1974). Stress waves generated in thin metallic films by a q-switched ruby laser, J. Appl. Phys., 45: 2601.

Zel 'dovich, Y. B., and Raizer, Y. P. (1965). Cascade ionization of a gas by a light pulse, Sov. Phys. JETP, 20: 772. 